\title{
Micro-macro correlations and anisotropy in granular assemblies under uniaxial loading and unloading
}

\author{
Olukayode I. Imole, ${ }^{*}$ Mateusz Wojtkowski, Vanessa Magnanimo, and Stefan Luding \\ Multi Scale Mechanics (MSM), Faculty of Engineering Technology, MESA+, University of Twente, \\ P.O. Box 217, 7500 AE Enschede, The Netherlands
}

(Received 30 October 2013; published 30 April 2014)

\begin{abstract}
The influence of contact friction on the behavior of dense, polydisperse granular assemblies under uniaxial (oedometric) loading and unloading deformation is studied using discrete element simulations. Even though the uniaxial deformation protocol is one of the "simplest" element tests possible, the evolution of the structural anisotropy necessitates its careful analysis and understanding, since it is the source of interesting and unexpected observations. On the macroscopic, homogenized, continuum scale, the deviatoric stress ratio and the deviatoric fabric, i.e., the microstructure behave in a different fashion during uniaxial loading and unloading. The maximal stress ratio and strain increase with increasing contact friction. In contrast, the deviatoric fabric reaches its maximum at a unique strain level independent of friction, with the maximal value decreasing with friction. For unloading, both stress and fabric respond to unloading strain with a friction-dependent delay but at different strains. On the micro-level, a friction-dependent non-symmetry of the proportion of weak (strong) and sliding (sticking) contacts with respect to the total contacts during loading and unloading is observed. Coupled to this, from the directional probability distribution, the "memory" and history-dependent behavior of granular systems is confirmed. Surprisingly, while a rank-2 tensor is sufficient to describe the evolution of the normal force directions, a sixth order harmonic approximation is necessary to describe the probability distribution of contacts, tangential force, and mobilized friction. We conclude that the simple uniaxial deformation activates microscopic phenomena not only in the active Cartesian directions, but also at intermediate orientations, with the tilt angle being dependent on friction, so that this microstructural features cause the interesting, nontrivial macroscopic behavior.
\end{abstract}

DOI: 10.1103/PhysRevE.89.042210

PACS number(s): 45.70.Cc, 81.05.Rm, 81.20.Ev

\section{INTRODUCTION AND BACKGROUND}

Granular materials are omnipresent in nature and widely used in various industries such as food, pharmaceutical, agriculture, and mining, among others. In many granular systems, interesting phenomena like dilatancy, anisotropy, shear-band localization, history dependence, jamming, and yield have attracted significant scientific interest over the past decade [1-3]. The bulk behavior of these materials depends on the behavior of their constituents (particles) interacting through contact forces. To understand their behavior, various laboratory element tests can be performed [4,5]. Element tests are (ideally homogeneous) macroscopic tests in which one can control the stress and/or strain path. Such macroscopic experiments are important ingredients in developing and calibrating constitutive relations, but they provide little information on the microscopic origin of the bulk flow behavior. An alternative is the discrete element method (DEM) [3], since it provides information about the micro-structure beyond what is experimentally accessible.

One element test which can easily be realized (experimentally or numerically) is the uniaxial (or oedometric) compression in a cylindrical or box geometry, involving an axial deformation of a bulk sample while the lateral boundaries of the system are fixed. During uniaxial loading, isotropic compression and nonisotropic deformation are superposed, so that both pressure and shear stress build up. After reversal,

\footnotetext{
*o.i.imole@utwente.nl
}

pressure and shear stress decay and the latter changes sign after a finite strain, which depends on friction. When a granular material is sheared, along with the shear stress, also anisotropy of the contact network begins to develop. Besides density and stress, anisotropy is an important ingredient to fully understand the micro-macro mechanics of granular materials.

In addition, the effects of contact friction between the constituent grains influence the micromechanical response under uniaxial loading, such that a rather simple element test begins to reveal interesting features. Several studies have numerically investigated the extent to which the response of granular media is affected by friction [6-10], especially in the triaxial geometry, but not many studies exist on uniaxial loading and unloading of frictional systems [11].

Also, the transmission of stress between contacts is relevant, as detailed in this study. Visualizations of the distribution of forces using photoelastic particles in two dimensions is about the only way to access this information experimentallysee $[12,13]$ and references therein-even though threedimensional (3D) photoelasticity and other neutron diffraction methods [14] have also been employed. Earlier numerical studies have highlighted the particular character of the contact force network, showing that strong contacts carrying force larger than the average are oriented anisotropically, with preferred direction parallel to the axis of compression, while those originating from weak contacts are isotropic or have a weak orientation orthogonal to the compression axis $[15,16]$. Another interesting issue is the distribution and orientation of tangential forces during the deformation of dense frictional packings [16-18]. In early, two-dimensional studies on 
frictional avalanching [17], it has been observed that friction is mobilized mostly from weak contacts, whereas strong contacts resist friction mobilization.

It is important at this point to distinguish between the three-dimensional uniaxial element test and the triaxial test. In the standard triaxial test, stress (or strain) is imposed on the sample in the axial (vertical) direction $\left(\sigma_{1}\right)$ while the stress in the lateral (horizontal) directions $\left(\sigma_{2}\right.$ and $\left.\sigma_{3}\right)$ are kept constant (i.e., $\sigma_{1} \neq \sigma_{2}=\sigma_{3}$ ). A striking difference between both tests is in the lateral direction where stress is kept constant in the triaxial test $\left(\sigma_{2}=\sigma_{3}=\sigma_{0}\right)$ but considerably increases from its initial value $\sigma_{0}$, in the uniaxial test where $\left(\sigma_{2} \approx \sigma_{3}\right)>\sigma_{0}$, since the walls are fixed. As with the uniaxial test, the stress in the axial direction is typically higher than the two lateral stresses during triaxial loading. Even though the difference in the boundary conditions has been shown to lead to different response [19], what has been less explored is the microscopic origin of the observed differences. This is surprising as oedometric (uniaxial) tests are also greatly relevant and widely used for the mechanical characterization and study of the consolidation properties of soils, as they reproduce field conditions. Thus, a deep understanding of the kinematics at particle scale in such a device is of great importance. It is also worth mentioning that the triaxial test is mostly used in geotechnical applications such as the testing of sands and rocks at very high stress levels. Since the broader focus of our research is the testing of frictional and cohesive granular materials for applications in the food, chemical, and agricultural industries, we focus on the much simpler uniaxial compression test where strong decrease in volume leads to compression and considerable increase in pressure and juxtapose our findings with those obtained in the triaxial test.

In the present study, we use discrete element simulations of confined uniaxial compression tests to investigate and relate the dependencies between the microscopic observations presented hereafter with the evolution of macroscopic quantities such as pressure and deviatoric stress-and to further extend this to explain the evolution of the structural or contact and force or stress anisotropies.

This work is structured as follows. We first describe the simulation method and model parameters along with the preparation and test procedures in Sec. II. The definitions of averaged micro-macro quantities including strain, stress, and structural anisotropies are presented in Sec. III. Where given, anisotropy refers to not only the deviatoric stress, but also to the direction dependence and inhomogeneity of forces, i.e., its microscopic origin. Next, we discuss the results of the current study by presenting the evolution of the stress and structural anisotropies during uniaxial loading and unloading in Sec. IV B followed by the magnitude and orientation of their respective eigenvalues in Sec. IV C. Furthermore, we discuss friction mobilization in Sec. IV E followed by the probability density functions of the normal and tangential forces in Sec. IV F and the classification of weak and strong forces in Sec. IV F. In Sec. V, we discuss the polar representation of the contact distribution based on the constant surface and constant bin width method and extract the structural anisotropy parameters using a sixth order Legendre spherical harmonic approximation in Sec. V A. Finally, the summary, conclusions, and outlook are presented in Sec. VI.
TABLE I. Summary and numerical values of particle parameters used in the DEM simulations, where $\mu$, the contact coefficient of friction, is varied in the following. For more details, see Ref. [3].

\begin{tabular}{lcl}
\hline \hline & Value unit & \multicolumn{1}{c}{ Description } \\
\hline$N$ & 9261 & Number of particles \\
$\langle r\rangle$ & $1(\mathrm{~mm})$ & Average radius \\
$w$ & 1.5 & Polydispersity $w=r_{\max } / r_{\min }$ \\
$\rho$ & $2000\left(\mathrm{~kg} / \mathrm{m}^{3}\right)$ & Particle density \\
$k_{n}$ & $10^{5}\left(\mathrm{~kg} / \mathrm{s}^{2}\right)$ & Normal spring stiffness \\
$k_{t}$ & $2.10^{4}\left(\mathrm{~kg} / \mathrm{s}^{2}\right)$ & Tangential spring stiffness \\
$\mu$ & $\mathrm{vary}$ & Coefficient of friction \\
$\gamma_{n}$ & $1000(\mathrm{~kg} / \mathrm{s})$ & Viscosity-normal direction \\
$\gamma_{t}$ & $200(\mathrm{~kg} / \mathrm{s})$ & Viscosity-tangential direction \\
$\gamma_{b t}$ & $100(\mathrm{~kg} / \mathrm{s})$ & Background damping-translational \\
$\gamma_{b r}$ & $20(\mathrm{~kg} / \mathrm{s})$ & Background damping-rotational \\
$t_{c}$ & $0.64(\mu \mathrm{s})$ & Contact duration (average) \\
\hline \hline
\end{tabular}

\section{SIMULATION DETAILS}

We use the discrete element method (DEM) [3] with a simple linear viscoelastic normal contact force law $f^{n} \hat{\mathbf{n}}=$ $(k \delta+\gamma \dot{\delta}) \hat{\mathbf{n}}$, where $k$ is the spring stiffness, $\gamma_{n}$ is the contact viscosity parameter, and $\delta$ or $\dot{\delta}$ are the overlap or the relative velocity in the normal direction $\hat{\mathbf{n}}$. The normal force is complemented by a tangential force law [3], such that the total force at contact $c$ is $\mathbf{f}_{c}=f_{n} \hat{\mathbf{n}}+f_{t} \hat{\mathbf{t}}$, where $\hat{\mathbf{n}} \cdot \hat{\mathbf{t}}=0$, with tangential force unit vector $\hat{\mathbf{t}}$. A summary of the values of the parameters used is shown in Table I, with sliding and sticking friction $\mu=\mu_{s l}=\mu_{s t}$ and rolling-and torsion-torques inactive $\left(\mu_{r}=\mu_{t}=0\right)$. An artificial viscous dissipation force proportional to the velocity of the particle is added for both translational and rotational degrees of freedom, resembling the damping due to a background medium, as, e.g., a fluid.

\section{A. Simulation setup and boundary conditions}

The simulation setup is a cuboid volume [20], triaxial box, with periodic boundaries on all sides. Since careful, welldefined sample preparation is essential, to obtain reproducible results [21], we follow a three-step procedure where friction is active in all the preparation stages:

(i) Spherical particles are randomly generated in the $3 \mathrm{D}$ box with low volume fraction and rather large random velocities, such that they have sufficient space and time to exchange places and to randomize themselves.

(ii) This granular gas is then isotropically compressed to a target volume fraction $v_{0}$ slightly below the jamming volume fraction.

(iii) This is followed by a relaxation period at constant volume fraction to allow the particles to dissipate their kinetic energy before further preparation or the actual element test is initiated.

\section{B. Isotropic compression methods}

After the three-step preparation, an isotropic compression test can be initiated to measure isotropic properties and to prepare further initial configurations at different volume fractions, with subsequent relaxation, so that we have a series 


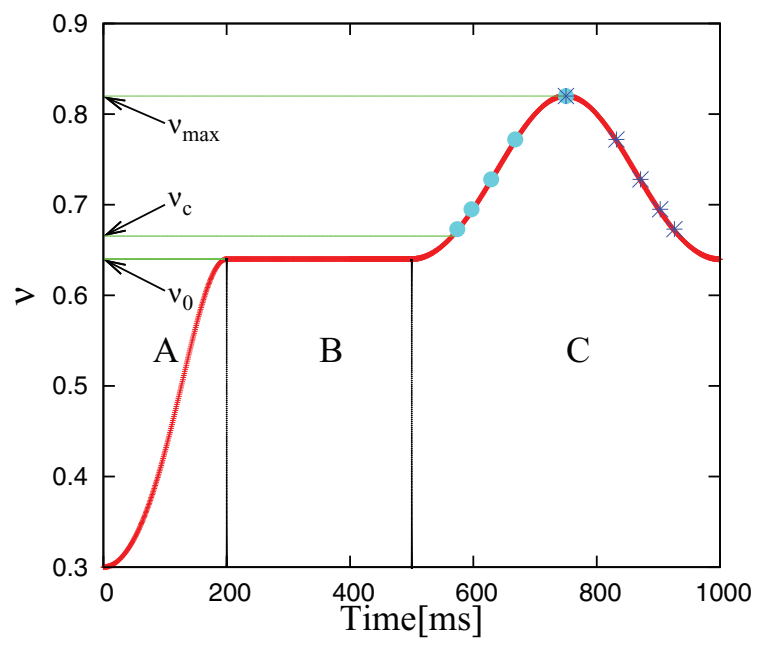

FIG. 1. (Color online) Evolution of volume fraction as a function of time. Region A represents the initial isotropic compression below the jamming volume fraction. B represents relaxation of the system to fully dissipate the systems potential and kinetic energy and $\mathrm{C}$ represents the subsequent isotropic compression up to $v_{\max }=0.820$ and then subsequent decompression. Cyan dots represent some of the initial configurations, at different $v_{i}$, during the loading cycle; blue stars, for the same $v_{i}$, are different configurations, since obtained during the unloading cycle; both can be chosen for further study.

of different reference isotropic configurations, achieved during loading and unloading, as displayed in Fig. 1. The goal here is to approach a direction independent isotropic configuration above the jamming volume fraction $v_{c}$, i.e., the transition point from fluidlike behavior to solidlike behavior [22]. Note that the initial packings for the respective frictional configurations are inherently different since they are prepared with the different friction coefficients active from the beginning of the first isotropic preparation stage (stage A in Fig. 1). We only keep as control parameter the volume fraction which is identical for the different configurations even though other micro-macro quantities such as pressure and coordination number will be different at a given volume fraction.

In the current study, to obtain a homogeneous initial isotropic configuration, several driving modes have been compared and these modes are discussed briefly in the following section. Later, for uniaxial tests, unless explicitly mentioned, the wall-driven uniaxial deformation protocol is applied. We tested the wall-driven against the strain-rate driven protocols for some quantities of interest to this work and realize that they lead to mostly the same results-besides some small details (see Sec. II B 5). Note that particular attention must be placed on the choice of the preparation protocol when other boundary conditions or quantities are considered as this conclusion may no longer hold. Even though strain-rate driven produces more homogeneous systems, we use the wall-driven mode since it more resembles the real experiment therefore important for future experimental validation of this work [23].

\section{Wall-driven isotropic compression}

In the first method, the periodic walls of the box are subjected to a strain-controlled motion following a co-sinusoidal law such that the position of, e.g., the top wall as a function of time $t$ is

$$
z(t)=z_{f}+\frac{z_{0}-z_{f}}{2}(1+\cos 2 \pi f t)
$$

with engineering strain

$$
\epsilon_{z z}(t)=1-\frac{z(t)}{z_{0}},
$$

where $z_{0}$ is the initial box length and $z_{f}$ is the box length at maximum strain, respectively, and $f=T^{-1}$ is the frequency. The maximum deformation is reached after half a period $t=T / 2$, and the maximum strain rate applied during the deformation is $\dot{\epsilon}_{z z}^{\max }=2 \pi f\left(z_{0}-z_{f}\right) /\left(2 z_{0}\right)=\pi f\left(z_{0}-z_{f}\right) / z_{0}$. The co-sinusoidal law allows for a smooth start-up and finish of the motion so that shocks and inertia effects are reduced. Also, the walls were driven in a quasistatic manner such that the ratio of the kinetic and potential energy is as low as possible $\left(E_{k} / E_{p} \leqslant 10^{-5}\right)$. By performing slower deformations, the energy ratio can be reduced even further [20].

\section{Pressure controlled isotropic deformation}

In the pressure controlled mode, the (virtual) periodic walls of the system are subjected to a co-sinusoidal periodic pressure control until the target pressure is achieved; for details see [24]. To achieve this, we set the mass of the virtual periodic walls of the system $m_{\mathrm{w}}$, to be of the order of the total mass of the particles in the system, leading to consistent behavior. The pressure controlled motion of the walls is described by [24]

$$
m_{\mathrm{w}} \ddot{x}_{\mathrm{w}}(t)=F_{\mathrm{x}}(t)-p A_{\mathrm{x}}(t)-\gamma_{\mathrm{w}} \dot{x}(t),
$$

where $F_{\mathrm{X}}(t)$ is the force due to the bulk material, $p A_{\mathrm{x}}(t)$ is the force related to the external load, and the last term is a viscous force, which damps the motion of the wall so that oscillations are reduced. $A_{\mathrm{x}}$ is the area of the wall perpendicular to $x$ where $x$ can be replaced by $y$ or $z$ in Eq. (3) for other walls. We find that large values of $m_{\mathrm{w}}$ generally lead to large energy fluctuations or oscillations while the final pressure is more rapidly approached for systems with smaller $m_{\mathrm{w}}$. In contrast, too small $m_{\mathrm{w}}$ can lead to violent motions and should be avoided. Additionally, we must mention that for our simulations, the sensitivity of the system to the wall dissipation is small since the simulations are performed in the very slow, quasistatic regime.

\section{Homogeneous strain-rate controlled isotropic deformation}

In this method, we apply a homogeneous strain rate to all particles in the ensemble and to the walls in each time step, such that each particle experiences an affine simultaneous displacement according to the diagonal strain rate tensor:

$$
\dot{\mathbf{E}}=\dot{\epsilon}_{\mathrm{v}}\left(\begin{array}{rrr}
-1 & 0 & 0 \\
0 & -1 & 0 \\
0 & 0 & -1
\end{array}\right),
$$

where $\dot{\epsilon}_{\mathrm{v}}(>0)$ is the rate amplitude applied until a target maximum volume fraction of, e.g., $v_{\max }=0.82$ is achieved. The DEM dynamics allows the particles to approach mechanical equilibrium by following the new unbalanced forces that lead 
to nonaffine displacements due to the new forces at each time step, or after a relaxation period.

\section{Swelling of particles}

An alternative isotropic deformation protocol is to allow the particle radii $r$ to slowly "grow" at rate $g_{r}$ from an initial volume fraction according to the relation $d r / d t=g_{r} r$. The swelling of the particles leads to a change in the volume fraction until the target volume fraction is achieved [25,26]. During the growth period, the particle mass changes with the radius. Additionally, the volume fraction also changes with time according to the relation $d v / d t=3 v g_{r}$, leading to the volume fraction $v=v_{0} \exp \left\{3 g_{r} t\right\}$ as a function of time $t$. The detailed form of the growth law with time is not relevant here, since all rates are very small.

\section{Comparison of driving modes}

In summary, comparing the preparation methods, we find that isotropic quantities like pressure, coordination number, or isotropic fabric evolve in a similar fashion for all driving modes. However, the strain-rate controlled isotropic preparation leads to very homogeneous configurations especially when viewed in terms of the mobilized friction. In the wall driven case, we find that friction is more highly mobilized in the contacts closest to the virtual periodic walls of the system leading to slight inhomogeneities. However, when the particles closest to the wall (up to $\approx 30 \%$ of the box length) are excluded from the computation, the resulting probability distributions as well as the field quantities show negligible differences with respect to the data from the full sample analysis. Due to this assessment, we choose here to focus on the wall driven isotropic compression since this more resembles experimental setups and is especially suitable for the subsequent uniaxial compression mode. Additionally, the co-sinusoidal wall motion allows for a smooth start-up and end of the compression cycle unlike the "kick" (even though tiny) to each particle in the strain rate controlled protocol. To be confident with our conclusions, some data are checked by comparing them with simulations performed with the strain-rate protocol, without coming to different conclusions.

\section{Uniaxial loading and unloading}

After isotropic compression, initial states can be chosen from the loading or unloading branch (after relaxation to allow for kinetic energy dissipation) from which the uniaxial test is initiated.

As an element test, uniaxial compression is achieved by moving the periodic walls in the $z$ direction according to a prescribed co-sinusoidal strain path [20], as shown in Eq. (1), with diagonal strain-rate tensor

$$
\dot{\mathbf{E}}=\dot{\epsilon}_{\mathrm{u}}\left(\begin{array}{rrr}
0 & 0 & 0 \\
0 & 0 & 0 \\
0 & 0 & -1
\end{array}\right)
$$

where $\dot{\epsilon}_{\mathrm{u}}$ is the strain rate (compression $>0$ and decompression/tension $<0$ ) amplitude applied in the uniaxial mode. The negative sign (convention) of $\dot{E}_{z z}$ corresponds to a reduction of length, so that tensile deformation is positive. During loading (compression) the volume fraction increases from $v_{0}$ (at dimensionless time $\tau=t / T_{\max }=0$ ) to a maximum $v_{\max }=0.820(\tau=0.5)$ and reverses back to the original $v_{0}$ at the end of the cycle $(\tau=1)$, after complete unloading. For more details on preparation and other parameters, see Ref. [20].

Even though the strain is imposed only on one mobile periodic "wall" with normal in the $z$ direction, which leads to an increase of compressive stress during compression, also the nonmobile $x$ and $y$ directions experience some stress increase as expected for "solid" materials with nonzero Poisson ratio, as discussed in more detail in the following sections.

However, during decompression the stress on the passive walls is typically smaller than that of the mobile, active wall, as consistent with anisotropic materials and findings from simulations and laboratory element tests using the biaxial tester [27,28] or the so-called lambdameter [29]. One of the main goals of this study is to also understand the behavior of the packing when compression is changed or reversed to tension.

\section{DEFINITIONS OF AVERAGED QUANTITIES}

In this section, we present the general definitions of averaged microscopic and macroscopic quantities.

\section{A. General tensor formulation}

To describe and better understand the relationships between macroscopic quantities, tensors are split up into isotropic, deviatoric, and antisymmetric parts. Each tensor can be decomposed as

$$
\boldsymbol{T}=\frac{1}{2}\left(\boldsymbol{T}+\boldsymbol{T}^{T}\right)+\frac{1}{2}\left(\boldsymbol{T}-\boldsymbol{T}^{T}\right)=\boldsymbol{T}_{\text {sym }}+\boldsymbol{T}_{\text {skew }},
$$

where $\boldsymbol{T}_{\text {sym }}$ and $\boldsymbol{T}_{\text {skew }}$ are the symmetric and antisymmetric parts of the tensor and the superscript $T$ stands for transpose. Since we will focus on the symmetric part, we further decompose $\boldsymbol{T}_{\text {sym }}$ uniquely into its spherical and deviatoric parts as

$$
\boldsymbol{T}=T_{\mathrm{v}} \boldsymbol{I}+\boldsymbol{T}_{D}
$$

with $T_{\mathrm{v}}=(1 / 3) \operatorname{tr}(\boldsymbol{T})$ and the traceless deviator $\boldsymbol{T}_{D}=\boldsymbol{T}-$ $T_{\mathrm{V}} \boldsymbol{I}$. The latter contains information about the eigensystem of $\boldsymbol{T}$, that is identical to the eigensystem of $\boldsymbol{T}_{D}$ itself.

Any (deviatoric) tensor can be transformed using a transformation matrix $\boldsymbol{R}$ to obtain its diagonal form:

$$
\boldsymbol{T}_{\mathrm{D}}^{\mathrm{eig}}=\left(\begin{array}{ccc}
T_{\mathrm{D}}^{(1)} & 0 & 0 \\
0 & T_{\mathrm{D}}^{(2)} & 0 \\
0 & 0 & T_{\mathrm{D}}^{(3)}
\end{array}\right)=\boldsymbol{R}^{T} \cdot \boldsymbol{T}_{D} \cdot \boldsymbol{R},
$$

$\boldsymbol{T}_{D}=T_{i}-T_{\mathrm{v}} / 3$, where $T_{i}$ 's are eigenvalues of $\boldsymbol{T}$. Also, $T_{\mathrm{D}}^{(1)}$, $T_{\mathrm{D}}^{(2)}$, and $T_{\mathrm{D}}^{(3)}$ are the eigenvalues sorted such that, as convention, $T_{\mathrm{D}}^{(1)} \geqslant T_{\mathrm{D}}^{(2)} \geqslant T_{\mathrm{D}}^{(3)} \cdot \boldsymbol{R}=\left(\hat{\boldsymbol{n}}_{1}, \hat{\boldsymbol{n}}_{2}, \hat{\boldsymbol{n}}_{3}\right)$ is the orthogonal transformation matrix, composed of the corresponding eigenvectors, which transforms $\boldsymbol{T}_{D}$ to its eigensystem. According to linear algebra, Eq. (6) can also be expressed as

$$
\boldsymbol{T}_{D} \cdot \hat{\boldsymbol{n}}_{\alpha}=T_{D}^{\alpha} \hat{\boldsymbol{n}}_{\alpha}
$$

with $T_{D}^{\alpha}$ and $\hat{\boldsymbol{n}}_{\alpha}$ the $\alpha$ eigenvalue and eigenvector of $\boldsymbol{T}_{D}$, respectively. The symbol "." represents the inner product of 
the tensor $\boldsymbol{T}_{D}$ and the vector $\hat{\boldsymbol{n}}_{\alpha}$ which leads to a vector parallel to $\hat{\boldsymbol{n}}_{\alpha}$.

In the following, we provide a consistent decomposition for strain, stress, and fabric tensors. We choose here to describe each tensor in terms of its isotropic part (first invariant of the tensor) and the second $\left(J_{2}\right)$ and third $\left(J_{3}\right)$ invariant of the deviatoric tensor:

$$
\begin{gathered}
J_{2}=\frac{1}{2}\left[\left(T_{\mathrm{D}}^{(1)}\right)^{2}+\left(T_{\mathrm{D}}^{(2)}\right)^{2}+\left(T_{\mathrm{D}}^{(3)}\right)^{2}\right], \\
J_{3}=\operatorname{det}\left(\boldsymbol{T}_{D}\right)=T_{\mathrm{D}}^{(1)} T_{\mathrm{D}}^{(2)} T_{\mathrm{D}}^{(3)} .
\end{gathered}
$$

$J_{3}$ can further be written as $J_{3}=T_{\mathrm{D}}^{(1)} T_{\mathrm{D}}^{(2)}\left(-T_{\mathrm{D}}^{(1)}-T_{\mathrm{D}}^{(2)}\right)$, since we are dealing with deviators.

\section{B. Strain}

For any deformation, the isotropic part of the infinitesimal strain tensor $\epsilon_{\mathrm{v}}$ (in contrast to the true strain $\varepsilon_{\mathrm{v}}$ ) is defined as

$$
\epsilon_{\mathrm{v}}=\dot{\epsilon}_{\mathrm{v}} d t=\frac{\epsilon_{x x}+\epsilon_{y y}+\epsilon_{z z}}{3}=\frac{1}{3} \operatorname{tr}(\mathbf{E})=\frac{1}{3} \operatorname{tr}(\dot{\mathbf{E}}) d t,
$$

where $\epsilon_{\alpha \alpha}=\dot{\epsilon}_{\alpha \alpha} d t$ with $\alpha \alpha=x x, y y$, and $z z$ as the diagonal elements of the strain tensor $\mathbf{E}$ in the Cartesian $x, y, z$ reference system. The integral of $3 \epsilon_{\mathrm{v}}$ denoted by $\varepsilon_{\mathrm{v}}=3 \int_{V_{0}}^{V} \epsilon_{\mathrm{v}}$ is the true or logarithmic strain, i.e., the volume change of the system, relative to the initial reference volume $V_{0}$ [30].

Several definitions are available in the literature [31] to define the deviatoric magnitude of the strain. Here, we use the objective definition of the deviatoric strain in terms of its eigenvalues $\epsilon_{\mathrm{d}}^{(1)}, \epsilon_{\mathrm{d}}^{(2)}$, and $\epsilon_{\mathrm{d}}^{(3)}$ which is independent of the sign convention.

The deviatoric strain is defined as

$$
\epsilon_{\mathrm{dev}}=\sqrt{\frac{\left(\epsilon_{d}^{(1)}-\epsilon_{d}^{(2)}\right)^{2}+\left(\epsilon_{d}^{(2)}-\epsilon_{d}^{(3)}\right)^{2}+\left(\epsilon_{d}^{(3)}-\epsilon_{d}^{(1)}\right)^{2}}{2}},
$$

where $\epsilon_{\mathrm{dev}} \geqslant 0$ is the magnitude of the deviatoric strain.

Note that the wall motion is strain controlled and the infinitesimal strain corresponds to the external applied strain. Hence the eigenvalues for the strain tensor are in the Cartesian coordinate system (thus no transformation is needed). For the purely isotropic strain, $\boldsymbol{\epsilon}^{\mathrm{ISO}}=\epsilon_{\mathrm{v}} \boldsymbol{I}$, with $\epsilon_{\mathrm{dev}}=0$, which is direction independent by definition. The corresponding shape factor for the deviatoric strain $\Lambda^{(-\epsilon)}$, is represented as the ratio $\Lambda^{(-\epsilon)}:=\epsilon_{d}^{(2)} / \epsilon_{d}^{(1)}$.

\section{Stress}

From the simulations, one can determine the stress tensor (compressive stress is positive as convention) components:

$$
\sigma_{\alpha \beta}=\frac{1}{V}\left(\sum_{p \in V} m^{p} v_{\alpha}^{p} v_{\beta}^{p}-\sum_{c \in V} f_{\alpha}^{c} l_{\beta}^{c}\right),
$$

with particle $p$, mass $m^{p}$, velocity $v^{p}$, contact $c$, force $f^{c}$, and branch vector $l^{c}$, while Greek letters represent components $x, y$, and $z[20,32]$. The first sum is the kinetic energy density tensor while the second involves the contact-force dyadic product with the branch vector. Averaging, smoothing, or coarse graining $[33,34]$ in the vicinity of the averaging volume $V$ weighted according to the vicinity is not applied in this study, since averages are taken over the total volume. Furthermore, since the data in this study are quasistatic, the first sum can mostly be neglected. The isotropic stress is denoted as hydrostatic pressure:

$$
p=\sigma_{\mathrm{v}}=\frac{1}{3} \operatorname{tr}(\boldsymbol{\sigma}) .
$$

As already mentioned, we will focus on the eigenvalues of the deviatoric stress tensor $\lambda_{i}^{s}=\sigma_{i}^{\mathrm{D}}=\sigma_{i}-p$, as defined in Sec. III A, with the principal directions being the same for $\sigma$ and $\sigma^{D}$. The (scalar) deviatoric stress for our 3D uniaxial simulations is

$$
\sigma_{\mathrm{dev}}=\sqrt{\frac{\left(\lambda_{1}^{s}-\lambda_{2}^{s}\right)^{2}+\left(\lambda_{1}^{s}-\lambda_{3}^{s}\right)^{2}+\left(\lambda_{2}^{s}-\lambda_{3}^{s}\right)^{2}}{2}} .
$$

The deviatoric stress ratio, $s_{\mathrm{dev}}=\sigma_{\mathrm{dev}} / p$, quantifies the "stress anisotropy"-where $\sigma_{\mathrm{dev}}=\sqrt{3 J_{2}^{\sigma}}$, with $J_{2}^{\sigma}$ the second invariant of the deviatoric stress tensor. The third stress invariant $J_{3}^{\sigma}=\lambda_{1}^{s} \lambda_{2}^{s} \lambda_{3}^{s}=\lambda_{1}^{s} \lambda_{2}^{s}\left(-\lambda_{1}^{s}-\lambda_{2}^{s}\right)=$ $\lambda_{1}^{s}\left[-\Lambda_{1}^{\sigma}-\left(\Lambda_{1}^{\sigma}\right)^{2}\right]$ can be replaced by the shape factor $\Lambda^{\sigma}:=$ $\lambda_{2}^{s} / \lambda_{1}^{s}$, which switches from +1 at maximum uniaxial loading to $-1 / 2$ after some unloading as will be shown below.

\section{Structural (fabric) anisotropy}

Besides the stress of a static packing of powders and grains, an important microscopic quantity of interest is the fabric or structure tensor. For disordered media, the concept of a fabric tensor naturally occurs when the system consists of an elastic network or a packing of discrete particles. A possible expression for the components of the fabric tensor is provided in Refs. [32,35]:

$$
F_{\alpha \beta}^{v}=\left\langle F^{p}\right\rangle=\frac{1}{V} \sum_{p \in V} V^{p} \sum_{c=1}^{N} n_{\alpha}^{c} n_{\beta}^{c},
$$

where $V^{p}$ is the particle volume of particle $p$ which lies inside the averaging volume $V$, and $n^{c}$ is the normal vector pointing from the center of particle $p$ to contact $c . F_{\alpha \beta}^{v}$ are thus the components of a symmetric rank two $3 \times 3$ tensor. In a large volume with some distribution of particle radii, the relationship between the trace of fabric, volume fraction $v$, and the average coordination number $C$ is given by $3 F_{\mathrm{v}}^{v}:=F_{\alpha \alpha}^{v}=g_{3} \nu C$, as reported in Ref. [36] and also confirmed from our wider friction $(\mu)$ data. The term $g_{3}$ corrects for the fact that the coordination number for different sized particles is proportional to their surface area such that for a monodisperse packing $g_{3}=1$ and for a polydisperse packing $g_{3}>1[30,35,37]$.

A different formulation for the fabric tensor considers simply the orientation of contacts normalized with the total number of contacts $N_{c}$, as follows [38-40]:

$$
F_{\alpha \beta}=\frac{1}{N_{c}} \sum_{c=1}^{N} n_{\alpha}^{c} n_{\beta}^{c},
$$

The relationship between Eqs. (15) and (16) is

$$
F_{\alpha \beta} \cong \frac{F_{\alpha \beta}^{v}}{g_{3} \nu C}=\frac{3 F_{\alpha \beta}^{v}}{F_{\mathrm{v}}},
$$

with the equality holding in the case of monodisperse systems. 
We can define the deviatoric tensor $\boldsymbol{F}^{\mathrm{D}}$ and calculate the eigenvalues $\lambda_{i}^{f}=F_{i}-F_{\mathrm{v}} / 3$ with $F_{\mathrm{v}}=1$, and $F_{i}$ the eigenvalues of the deviatoric fabric based on Eq. (16). We assume that the structural anisotropy in the system is quantified (completely) by the anisotropy of fabric, i.e., the deviatoric fabric, with scalar magnitude similar to Eqs. (11) and (14) as

$$
F_{\mathrm{dev}}=\sqrt{\frac{\left(\lambda_{1}^{f}-\lambda_{2}^{f}\right)^{2}+\left(\lambda_{1}^{f}-\lambda_{3}^{f}\right)^{2}+\left(\lambda_{2}^{f}-\lambda_{3}^{f}\right)^{2}}{2}},
$$

proportional to the second invariant of $\boldsymbol{F}^{\mathrm{D}}, F_{\mathrm{dev}}=\sqrt{3 J_{2}^{F}}$, where $\lambda_{1}^{f}, \lambda_{2}^{f}$, and $\lambda_{3}^{f}$ are the three eigenvalues of the deviatoric fabric tensor.

Alternatively, a simpler definition of the deviatoric fabric for an axial symmetric element test takes into account the difference between the fabric eigenvalue of the main compressive (axial) direction and the average values in the isotropic plane as follows:

$$
F_{\mathrm{dev}}^{*}=\lambda_{1}^{f}-\frac{\lambda_{2}^{f}+\lambda_{3}^{f}}{2} .
$$

Note that if $\lambda_{2}^{f}=\lambda_{3}^{f}$, Eqs. (18) and (19) coincide. Analogous to Eqs. (18) and (19), $F_{\mathrm{dev}}$ and $F_{\mathrm{dev}}^{*}$ can also be described using the definition of fabric presented in Eq. (15).

\section{E. Eigenvector orientation}

Due to the axial symmetry of the uniaxial compression mode, the orientation of the eigenvectors of stress and fabric can be defined with reference to the main compressive $z$ direction as

$$
\theta_{\alpha}=\arccos \left(\hat{\boldsymbol{n}}^{(\alpha)} \cdot \hat{z}\right),
$$

where $\hat{z}$ is the unit vector in the $z$ direction. Additionally, orientations are projected such that they lie within the range to $\pi / 2$.

\section{RESULTS AND OBSERVATIONS}

In this section, as results of the current study, first we will discuss the influence of friction on the evolution of stress and structural anisotropy as functions of deviatoric strain during loading and unloading. To complement these results, we investigate the magnitude and orientation of the eigenvalues of stress and fabric during loading and unloading and their respective shape factors. To gain insights into the relationship between the normal and tangential force on the macroscopic stress and structure, we report briefly their probability density functions (pdfs) for different frictional systems, as well as the force intensity weighted by the contact state. Finally, we present a sixth order harmonic approximation of the polar representation of contacts and forces to describe the axial-symmetric structural anisotropy, relating fabric to the pdfs.

\section{A. Pressure and coordination number}

Isotropic quantities during loading and unloading for various deformation paths were presented in Ref. [20] for frictionless particles and in Ref. [41] for frictional particles and will not be discussed in detail here. We only note that the coordination number and the hydrostatic pressure scale quantitatively differently with isotropic strain but behave in a very similar fashion in the cases of isotropic, pure deviatoric and uniaxial compression. The effects of polydispersity on the evolution of the isotropic quantities have also been extensively studied in Ref. [42] for various deformation paths. The isotropic quantities, namely pressure, coordination number, and fraction of rattlers, show a systematic dependence on the deformation mode and polydispersity via the respective jamming volume fractions. In addition, the pressure is coupled to the deviatoric strain via the structural anisotropy, as discussed in the next subsection.

Our uniaxial test starts from an initial volume fraction $v_{i}=0.692$ above the jamming volume fraction and reaches a maximum volume fraction $v_{\max }=0.82$ during loading before returning to $v_{i}$ for unloading. In Figs. 2(a) and 2(b), we plot the nondimensional pressure $p$ for different friction coefficients $\mu=0$ to 1 during loading and unloading, respectively. Here we define the nondimensional pressure as

$$
p=\frac{2\langle r\rangle}{3 k_{n}} \operatorname{tr}(\sigma),
$$

where $\operatorname{tr}(\boldsymbol{\sigma})$ is the trace of the stress tensor. The loading and unloading branches are close to the unloading branch having a tiny shift to the right due to hysteretic effects [30]. We observe that even though the different initial configurations are identical with respect to the initial volume fraction, their initial pressure states are different since their friction coefficients are activated right from the initial preparation stage (as in material being filled into a constant volume sample holder). An increase in $p$ with increasing $\mu$ is observed. Also, $p$ increases with increasing $v$ during uniaxial loading for all friction coefficients and for any given volume fraction. Extrapolating the pressure data towards smaller $v$ to $p \rightarrow 0$ leads to the respective jamming densities $v_{c}(\mu)$, which decrease with $\mu$ increasing [41]. Higher friction leads to more strongly compacted packings, since jamming sets in at lower densities, relative to the target density $v_{i}$. The initial states (with constant $v_{i}$ ) are the basis of the sometimes counterintuitive behavior, observed in the uniaxial tests below.

Furthermore, in Figs. 2(c) and 2(d), we plot the evolution of the coordination number $C^{*}$ as function of the volume fraction $v$ and show its dependence on friction during loading and unloading, respectively. The coordination number here is defined as the average number of contacts per particle in the ensemble. Here, we exclude the particles with less than four contacts (called rattlers) since they do not contribute to the mechanical stability of the packing [20,30,42]. During loading, we observe an increase in the coordination number followed by a decrease after strain reversal. We observe a systematic decrease in the coordination number with friction with the largest friction showing the smallest coordination number. This indicates that fewer contacts are necessary for stability with increasing friction, even though $p$ is larger. For both $p$ and $C^{*}$, the we observe a decreasing slope with friction.

In the following sections, we will focus on the nonisotropic quantities and their evolution with respect to the deviatoric strain. 


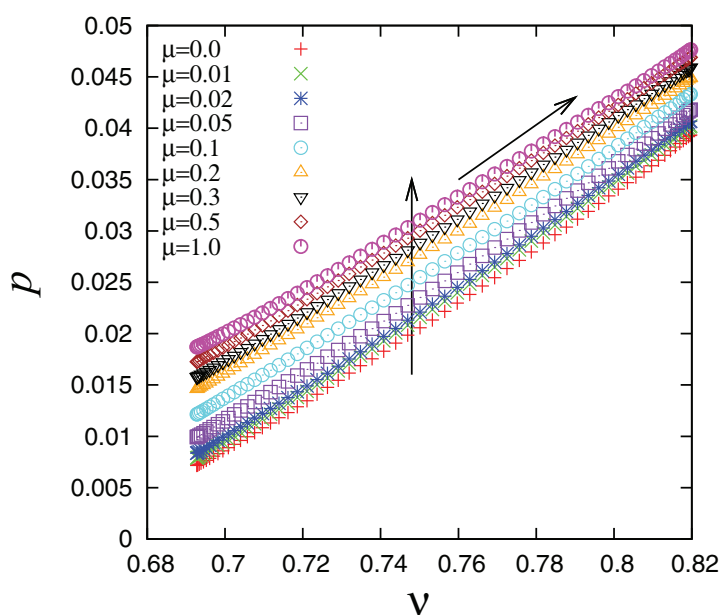

(a)

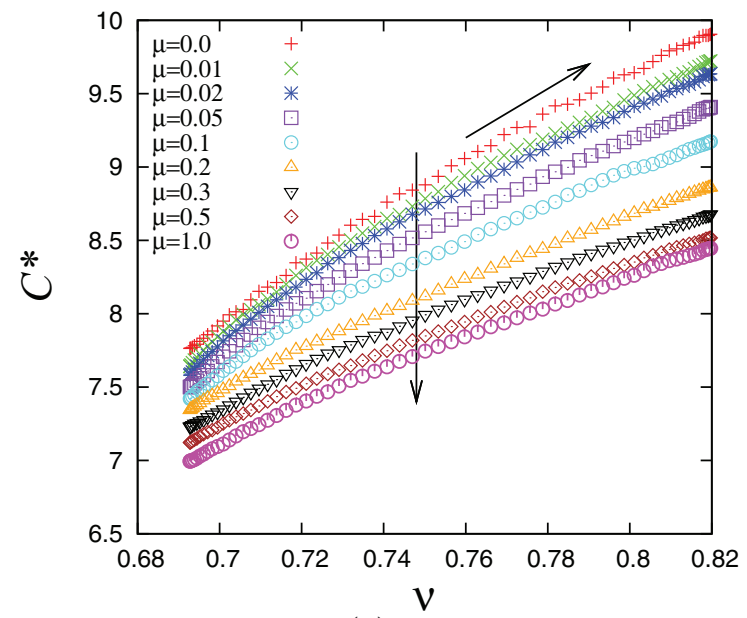

(c)

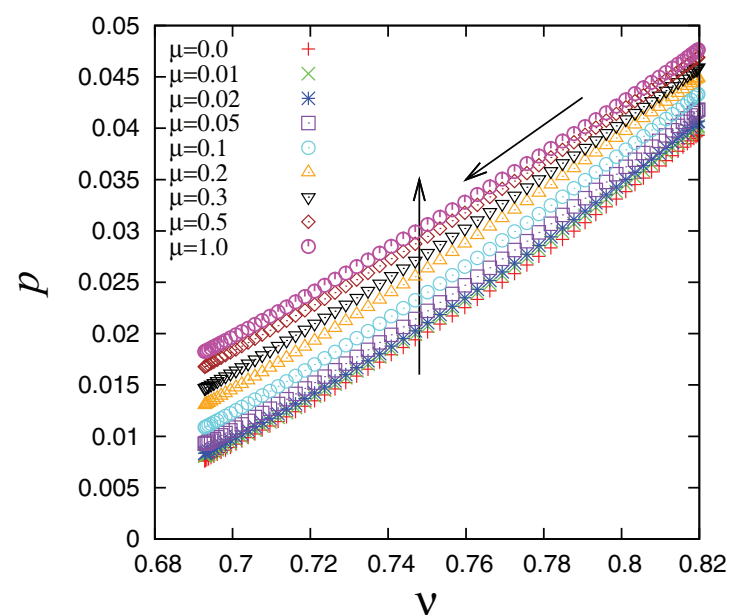

(b)

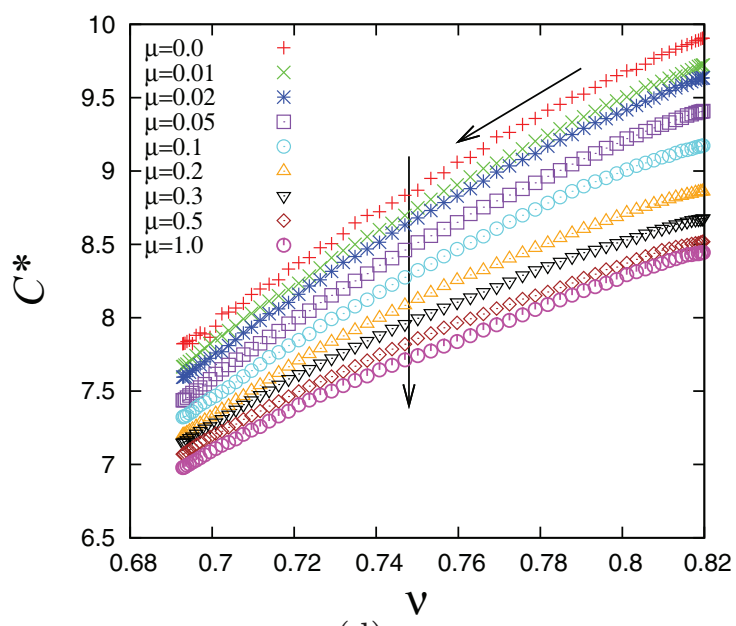

(d)

FIG. 2. (Color online) The nondimensional pressure plotted as function of volume fraction under uniaxial deformation for different friction coefficients during (a) loading and (b) unloading and coordination number (excluding rattlers) for the same dataset during (a) loading and (b) unloading. The vertical arrows show increasing (and decreasing) $\mu$ while the tilted arrows show the loading and unloading direction.

\section{B. Deviatoric stress and fabric}

Under uniaxial compression, shear stress, anisotropy of the contact and force networks develop, related to the creation and destruction of new contacts [20]. We term the deviatoric part of the stress tensor and its microscopic force-direction dependence as the "stress anisotropy," in parallel to the contact direction dependency of the structural anisotropy.

The deviatoric stress ratio $s_{\mathrm{dev}}=\sigma_{\mathrm{dev}} / p$ is shown in Figs. 3(a) and 3(b) for a frictionless $(\mu=0)$ and several frictional $(\mu=0.01,0.02,0.05,0.1,0.2,0.3,0.5$, and 1.0$)$ systems during uniaxial loading and unloading, respectively. As the deviatoric strain applied to the system is increased during uniaxial loading, the deviatoric stress ratio initially grows for all the friction coefficients shown. In some cases (for small $\mu$ ), the maximal $s_{\mathrm{dev}}$ is reached before the maximum deviatoric strain applied $\left(\varepsilon_{\mathrm{dev}}^{\max }=0.1549\right)$ is reached. For some of the configurations studied, an asymptote (or steady state) is observed in which further application of deviatoric strain does not lead to visible further increase or decrease in the deviatoric stress. At the maximum applied deviatoric strain, we observe that not all configurations (especially the highest friction coefficients) have reached full saturation. For the systems with lower microscopic friction coefficients, a slight decrease of the deviatoric stress ratio for larger deviatoric strains is seen. The slope of the deviatoric stress ratio, which represents its growth rate, shows a decreasing trend with increasing friction. Recall that the initial packings are different since they are prepared with different friction coefficients. Due to this, the pressure increases with increasing friction while the coordination number decreases with friction. The slope of the deviatoric stress ratio in Fig. 3(a), related to the initial shear stiffness of the isotropic packing, is proportional to these two quantities [43-45].

The evolution of the deviatoric stress during unloading (after strain reversal) is presented in Fig. 3(b). Note that due to the square-root definition used in Eq. (14), the deviatoric stress remains positive. ${ }^{1}$ During deviatoric unloading, $s_{\mathrm{dev}}$

\footnotetext{
${ }^{1}$ An alternative way to enforce the sign convention is to multiply the deviatoric stress Eq. (14) by the sign of the difference between the eigenvalue of the main compressive direction and the average in the other two fixed directions as given for fabric in Eq. (19). This leads
} 


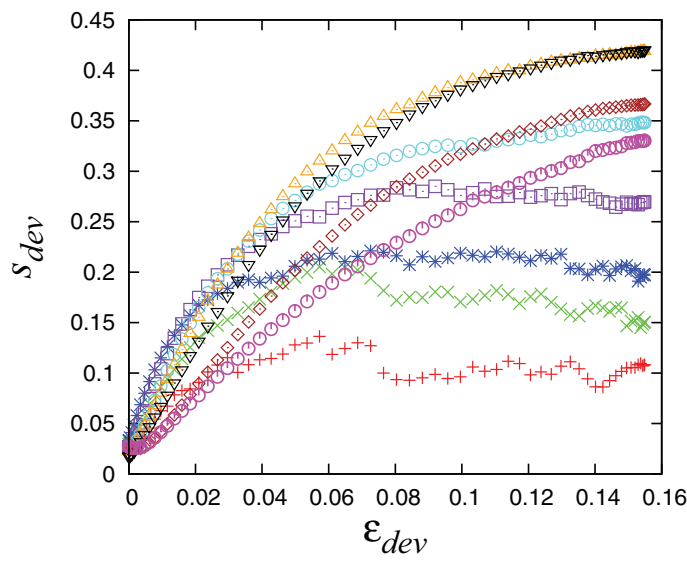

(a)

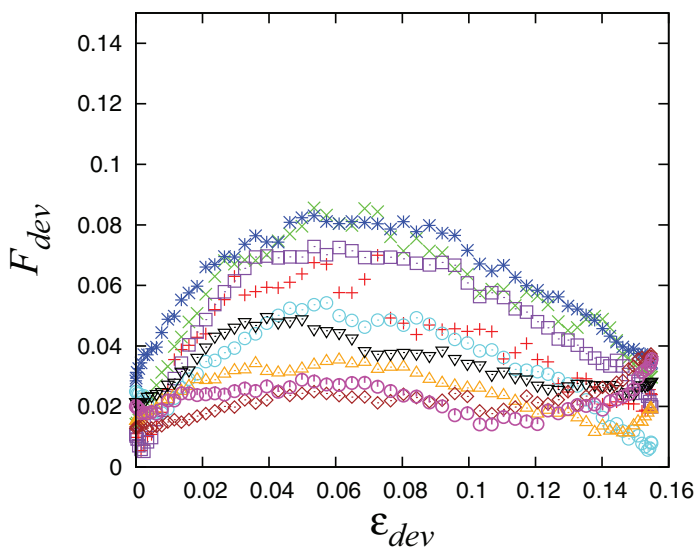

(c)

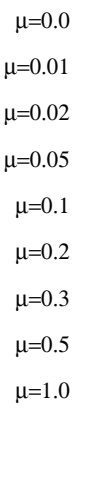
$\mu=0.01$ $\mu=0.02$ $\mu=0.05$ $\mu=0.1$ $\mu=0.2$ $\mu=0.3 \quad \nabla$ $\mu=0.5$ $\mu=1.0$

$$
\begin{aligned}
& + \\
& \times \\
& * \\
& \square \\
& \circ \\
& \triangle \\
& \nabla \\
& \diamond \\
& \odot
\end{aligned}
$$$$
\text { (1) }
$$

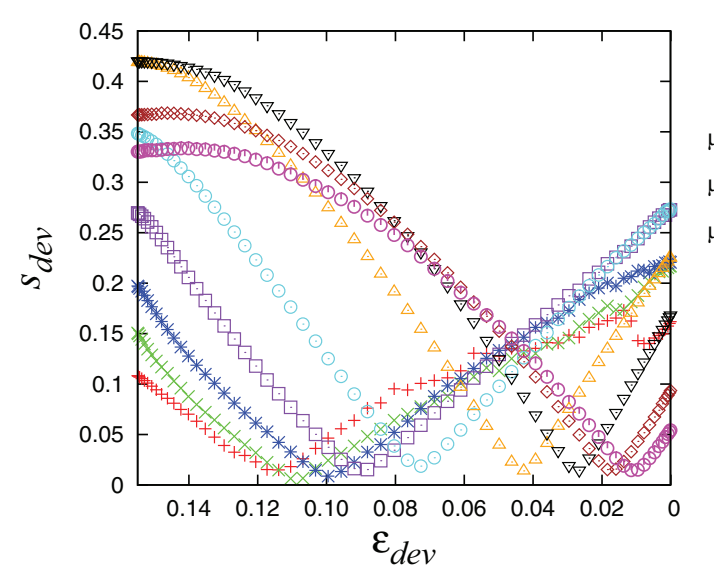

(b)

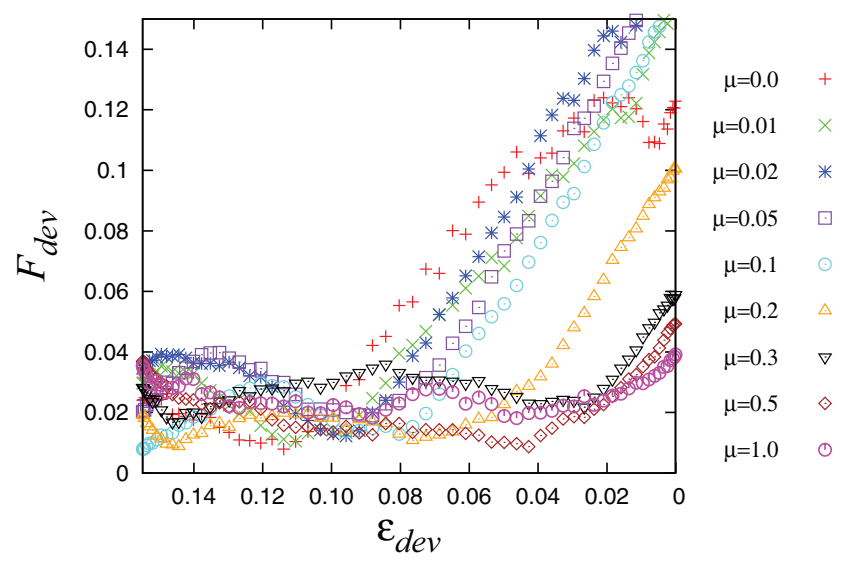

FIG. 3. (Color online) The deviatoric stress ratio plotted as a function of deviatoric strain during uniaxial (a) loading and (b) unloading. The corresponding plots of the deviatoric fabric plotted during uniaxial (c) loading and (d) unloading, for different microscopic friction coefficients.

begins to decrease until the system approaches an isotropic stress configuration, where $s_{\mathrm{dev}}=0$. The $\varepsilon_{\mathrm{dev}}$ values where $s_{\mathrm{dev}} \approx 0$ consistently decrease with increasing friction-as is consistent with the trend of the maximum $s_{\mathrm{dev}}$ values reached during uniaxial loading at larger $\varepsilon_{\mathrm{dev}}$ for stronger friction. For systems with large friction coefficients $(\mu=0.3,0.5$, and 1.0), the $\varepsilon_{\mathrm{dev}}$ values at which $s_{\mathrm{dev}}=0$ are closer to each other than for weakly frictional systems-see Fig. 9 below.

Along with the deviatoric stress ratio, for a characterization of the contact network, we plot the deviatoric fabric magnitudes $F_{\text {dev }}$ [as defined in Eq. (18)] of the systems discussed above as a function of the deviatoric strain during uniaxial loading and unloading in Figs. 3(c) and 3(d), respectively. In Fig. 3(c), the deviatoric fabric magnitude builds up from different (random, but small) initial values and reaches different maxima within the same range of deviatoric strain $\left(\varepsilon_{\mathrm{dev}} \approx 4-6 \%\right)$. For larger strains, we observe a decrease in the structural anisotropy towards zero. This is explained by the fact that more contacts are created in the axial compressive direction compared to the horizontal plane at the beginning of

to positive and negative $s_{\mathrm{dev}}$, which should take care of the strain reversal [46]. the loading cycle. At the maximum $\left(\varepsilon_{\mathrm{dev}} \approx 0.06\right)$, the material behavior changes such that the number of contacts created in the horizontal plane becomes higher with respect to the vertical plane. This interesting behavior will be further discussed when we analyze the magnitude and orientation of the respective eigenvectors in Sec. IV C.

After strain reversal, as presented in Fig. 3(d), the initial isotropic state is not recovered-a clear signature of history dependence and structural anisotropy being independent of (or decoupled from) the deviatoric stress ratio. Additionally, a strong difference can be seen in the fabric response of systems with lower and higher friction, respectively. As we will see later, the orientation of the eigenvalues of these systems provide interesting insights into these observations.

In general, comparing the evolution of deviatoric stress ratio and deviatoric fabric, we observe a strongly nonlinear qualitative even behavior though a linear contact model used. This confirms that the nonlinearity observed is due to the structural reorganization in the packing.

In Fig. 4, we plot the maximum deviatoric stress ratio and maximum deviatoric fabric reached from Figs. 3(a) and 3(c) for the respective friction coefficients. Interestingly, the maximum deviatoric stress ratios increase with increasing friction coefficient until $\mu \approx 0.25$, where it peaks at $s_{\mathrm{dev}}^{\max } \approx 0.43$ 


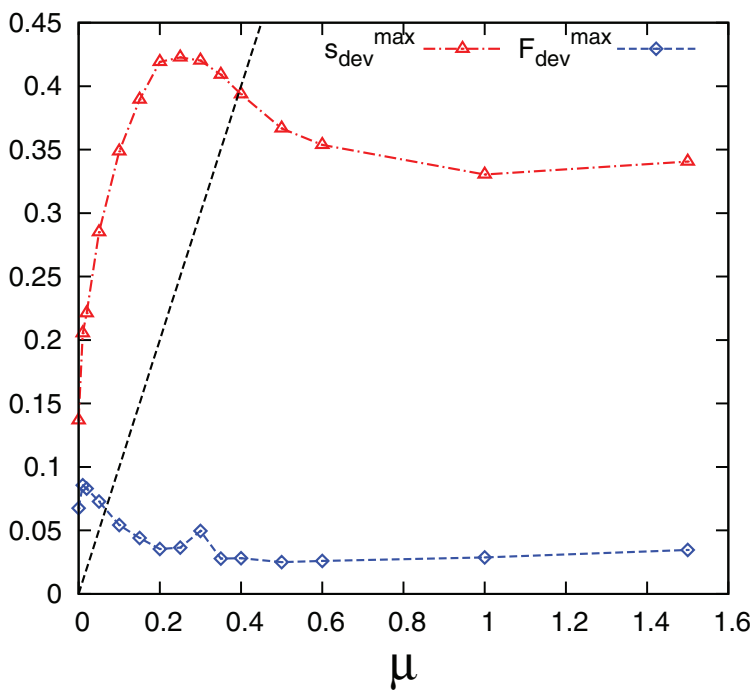

FIG. 4. (Color online) Trend of the peak deviatoric stress and peak deviatoric fabric with increasing microscopic friction coefficient $\mu$ under uniaxial loading, given a maximal strain $\varepsilon_{\mathrm{dev}}^{\max }=0.1549 . s_{\mathrm{dev}}^{\max }$ values for $\mu>0.1$ are taken at $\varepsilon_{\operatorname{dev}}^{\max }$ since no clear maximum is achieved. Dashed line indicates $\mu^{\text {macro }}=\mu$.

and subsequently decrease for higher friction coefficients. From Fig. 3(a) we observe that the highest friction coefficients (between $\mu=0.1$ and 1.0) appear not to have reached a final saturation; the application of further strain could lead to a higher maximum deviatoric stress ratio. Due to this, the decrease in the maximum deviatoric stress ratio at higher friction coefficients under uniaxial compression requires further attention. For our system where we control volume, we argue that at a maximum volume fraction $v_{\max }=0.82$, we are already close to the upper limit for realistic deformations with about $5 \%$ average overlaps, i.e., compression is very strong. Note that the maximum deviatoric stress ratio reached is termed the "macroscopic friction coefficient," $\mu^{\text {macro }}:=s_{\operatorname{dev}}^{\max }[20]$, representing the macroscopic mobilized friction. We note that the maxima reached are higher than the microscopic friction coefficient for systems with low friction, between $\mu=0$ and 0.4 , while for higher friction, the maxima are lower [47].

In Fig. 4, we also show the trend of the maximum structural anisotropy reached, $F_{\text {dev }}^{\max }$, with increasing friction. Besides for $\mu=0$, the maximum deviatoric fabric shows a decreasing trend with increasing friction and saturates at $F_{\mathrm{dev}}^{\max } \approx 0.025$ for the highest friction coefficients. In comparison, the structural anisotropy is much smaller than the deviatoric stress ratio. The decrease in the maximal structural anisotropy is in disagreement with observations reported for triaxial tests $[8,48]$, where it is observed to increase with increasing friction. One main reason is that under triaxial loading, the coordination number decreases with increasing strain (dilatancy), but it increases under uniaxial loading (due to ongoing compaction) while in both cases fabric anisotropy is induced by shearing. The second reason is the stronger compaction established initially for increasing $\mu$. For our system and preparation procedure, at a given density, the distance from the jamming point increases with increasing friction. The maximum fabric anisotropy decreases as the distance from the jamming volume fraction increases [20].

\section{Eigenvalues and eigenvectors of stress and fabric}

In this section, we will discuss the magnitude of the eigenvalues of deviatoric stress and deviatoric fabric during uniaxial loading and unloading as well as the orientation of the eigenvectors. As reference and representative example, we will show the data for only one of the coefficients of friction ( $\mu=0.1$ ) and discuss in words the interesting trends for the others. Finally, we will couple the observations to the evolution of stress and structural anisotropies presented in Sec. IV B.

In Figs. 5(a) and 5(b), we plot the eigenvalues of the deviatoric stress for the frictional system with $\mu=0.1$ during loading and unloading against deviatoric strain $\varepsilon_{\mathrm{dev}}$. During loading, $\lambda_{1}^{s}$, which corresponds to the stress eigenvalue of the axial compression direction, increases linearly from 0 and remains positive while the eigenvalues $\lambda_{2}^{s}$ and $\lambda_{3}^{s}$ of the two nonmobile directions are negative and very similar in magnitude. During unloading, $\lambda_{1}^{s}$ decreases but remains positive; at $\varepsilon_{\mathrm{dev}} \approx 0.075$, all eigenvalues become zero and then switch order, so that the axial direction eigenvalue becomes increasingly negative. The intermediate $\lambda_{2}^{s}$ then becomes identical to $\lambda_{1}^{s}$, both growing to positive values. After strain reversal, $\lambda_{1}^{s}$ returns along a different path, visible from the difference in slope before and after strain reversal. The orientation of the corresponding eigenvectors during loading and unloading are shown in Figs. 5(c) and 5(d). At $\varepsilon_{\mathrm{dev}}=0$, the orientations are different and random which is an indication of the almost isotropic initial configuration. With increasing strain, $\theta_{1}^{s}$, which corresponds to the orientation of the compressive stress eigenvalue, converges to $\theta^{\mathrm{s}}=0^{\circ}$ and remains until the end of the loading path. During this period, the stress and strain eigenvectors are said to be colinear with respect to each other. On the other hand, the orientation $\theta_{2}^{s}$ and $\theta_{3}^{s}$ of the other eigenvalues also drops to $\theta^{\mathrm{s}}=90^{\circ}$ showing a perpendicular alignment with respect to the compression direction. After strain reversal, the eigendirections of stress do not instantaneously respond to the directional change until $\varepsilon_{\mathrm{dev}} \approx 0.10$ where $\theta_{1}^{s}$ begins to increase to $90^{\circ}$ and finally reaches $\varepsilon_{\mathrm{dev}} \approx 0.03$. Accordingly, $\theta_{3}^{s}$ drops to $0^{\circ}$, while $\theta_{2}^{s}$ remains close to $90^{\circ}$ all the time.

The corresponding eigenvalue and eigenvector orientations of the deviatoric fabric for $\mu=0.1$ are presented in Figs. 6(a) and 6 (b) during uniaxial loading and unloading. Similar to the eigenvalues of stress, the major eigenvalue $\lambda_{1}^{f}$, remains positive while the two lower eigenvalues are negative. In contrast to stress, $\lambda_{1}^{f}$ increases and reaches a peak at $\varepsilon_{\mathrm{dev}} \approx$ 0.05 after which it begins to decrease towards zero as the maximum strain is approached. Also, $\lambda_{2}^{f}$ and $\lambda_{3}^{f}$ are not identical, i.e., $\lambda_{3}^{f}$ has a slightly higher magnitude than $\lambda_{2}^{f}$. This is an indication of the existence of anisotropy in the plane perpendicular to $\lambda_{1}^{f}$ even though the stress picture shows isotropy. At maximum deviatoric strain, however, the magnitudes of all the eigenvalues are close to zero. After strain reversal, $\lambda_{1}^{f}$ and $\lambda_{2}^{f}$ show an increasingly positive trend from $\varepsilon_{\mathrm{dev}} \approx 0.08$ but are not exactly identical in magnitude while $\lambda_{1}^{f}$ is negative and consistently decreases from $\varepsilon_{\mathrm{dev}} \approx$ 0.08 until the end of the decompression cycle. We observe however that immediately after strain reversal, $\lambda_{1}^{f}$ returns with the same slope as the loading [indicated by the black dotted lines in Figs. 6(a) and 6(b)] before finally changing 


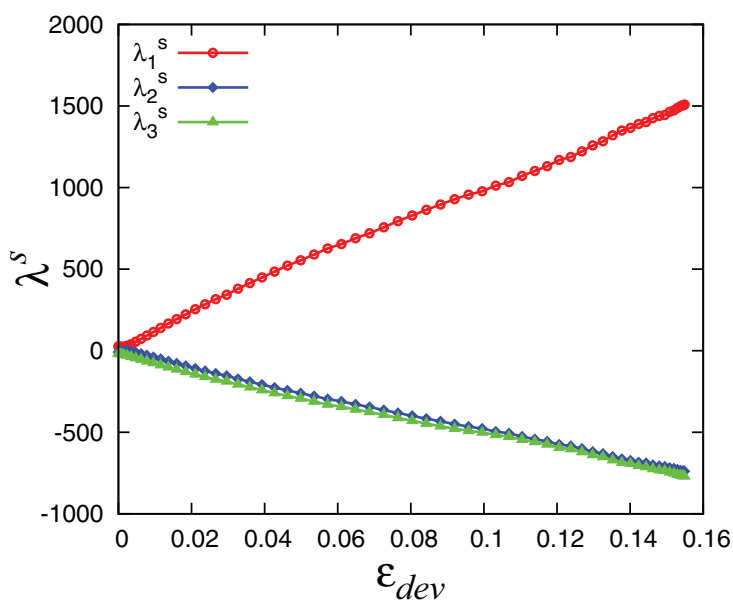

(a)

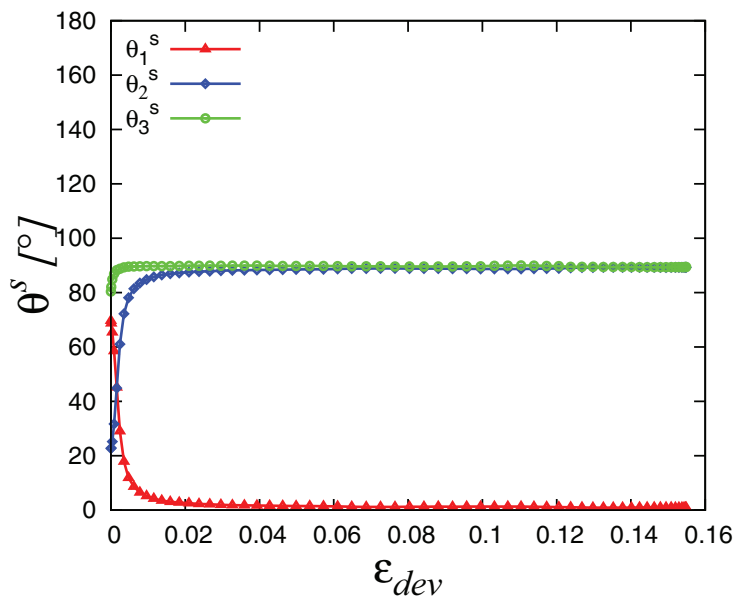

(c)

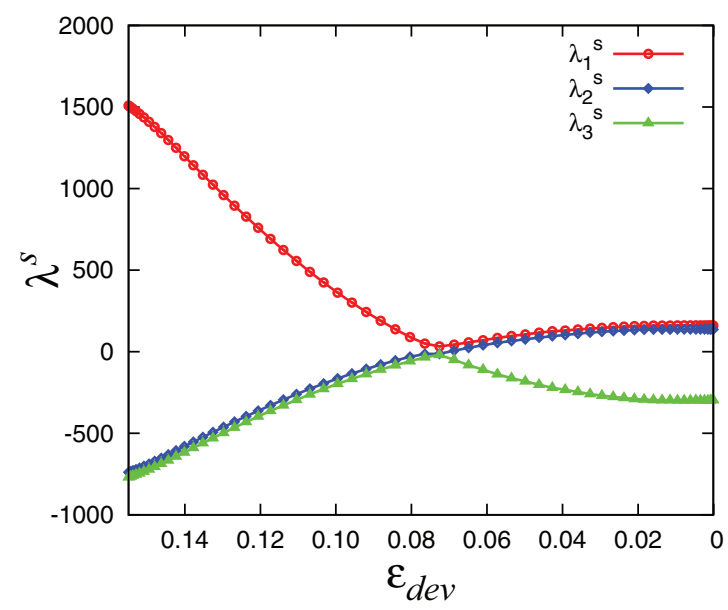

(b)

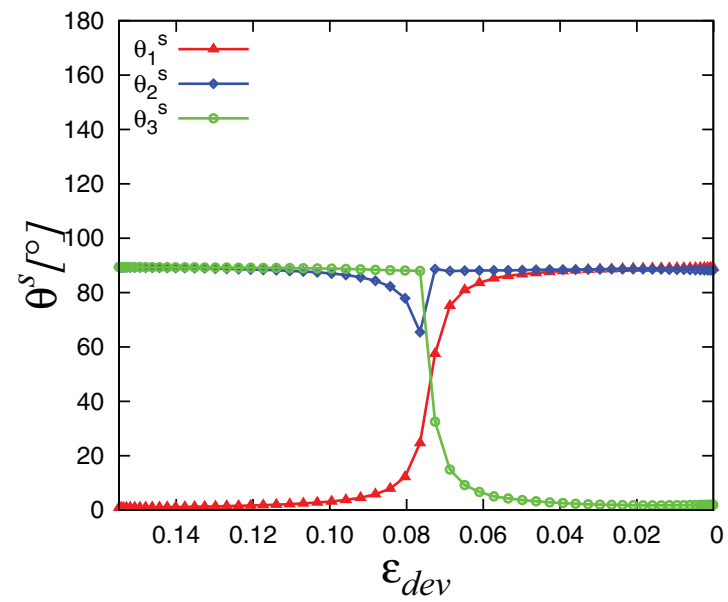

(d)

FIG. 5. (Color online) Eigenvalues of deviatoric stress for $\mu=0.1$ plotted as functions of the deviatoric strain for (a) loading and (b) unloading along with their corresponding orientations with respect to the compressive direction during uniaxial (c) loading and (d) unloading.

direction. When viewed in terms of the opening and closing of contacts, this indicates that immediately after strain reversal, contacts that just closed (mainly in the horizontal direction) re-open, leading to the initial increase in $\lambda_{1}^{f}$ along the same path. With further unloading, more contacts are lost in the vertical direction. With increasing friction, we observe that the reversible range increases leading to longer delays before the system responds actively to strain reversal deviating from such a trend. In general, we conclude that the response of stress and fabric to strain reversal are very different with respect to each other.

Similar to the stress, the orientations of the fabric components are interesting. Starting from random values, $\theta_{1}^{f}$ decreases and is close but distinct from $0^{\circ}$ during loading, while $\theta_{2}^{f}$ and $\theta_{3}^{f}$ are close to $90^{\circ}$ during the same period. This indicates that $\theta_{1}^{f}$ is not fully aligned with the strain eigenvector with the deviation showing the noncolinearity. After strain reversal, a delay can be seen before $\theta_{1}^{f}$ and $\theta_{3}^{f}$ transit to $90^{\circ}$ and $0^{\circ}$, respectively, while $\theta_{2}^{f}$ remains close to $90^{\circ}$.

Additionally, to fully describe the tensors, one can calculate the respective shape factors for stress and fabric, respectively, as the ratio of the eigenvalues as shown in Table II at the initial, maximum, and end of the uniaxial compressiondecompression cycle.

In the following analysis, we will investigate how the orientation changes with increasing the microscopic friction coefficient and the relationships with the force network.

In Figs. 8(a) and 8(b), we plot the orientations of the first eigenvectors of stress $\theta_{1}^{s}$ and fabric $\theta_{1}^{f}$ for all contacts and different friction coefficients, respectively. The initial value of $\theta_{1}^{s}$ is random at the beginning of the loading path for the different friction coefficients. As loading begins, $\theta_{1}^{s}$ decreases and at $\varepsilon_{\mathrm{dev}} \approx 0.02, \theta_{1}^{s} \approx 0^{\circ}$ for all friction. The relaxation rate (data scaled with the initial value of the respective $\theta_{1}^{s}$ ), shown as an inset on a log scale, is nonsystematic for the different friction coefficients possibly due to the initial isotropic configuration. Note that the angle $\theta_{1}^{s}$ does not exactly decrease to zero since $\theta_{1}^{s}$ is always positive even though it fluctuates around zero. Observing the behavior of the eigenvectors, we find that during loading, they approach zero (aligned with the compression direction) and remain until maximum compression. A slight delay is seen before the vectors finally flip back to the plane [49].After strain reversal at $\varepsilon_{\mathrm{dev}}=0.16$, the response of $\theta_{1(s)}$ is slow and it only begins to increase at $\varepsilon_{\mathrm{dev}} \approx 0.12$ for $\mu=0$. It is interesting to note that the delay time increases with 


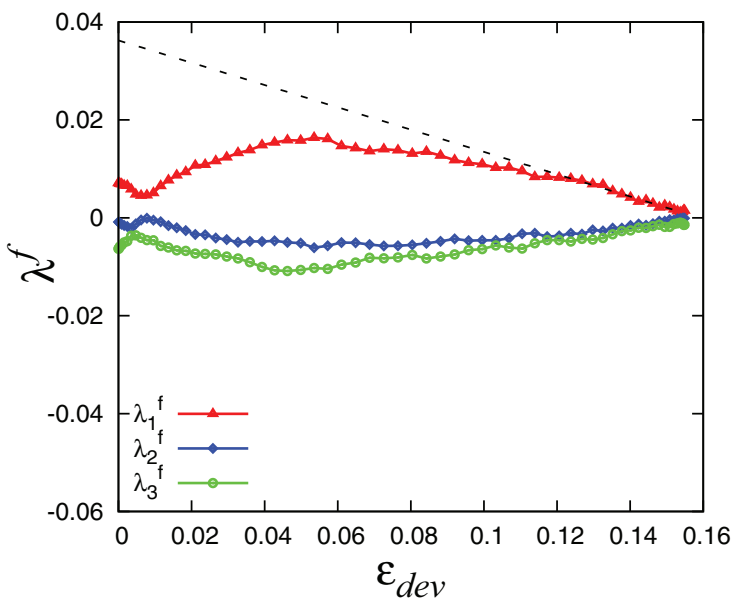

(a)

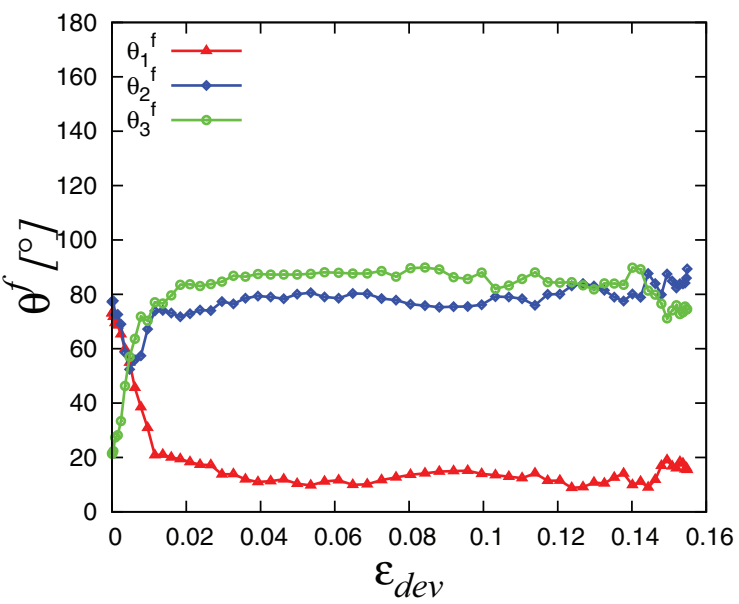

(c)

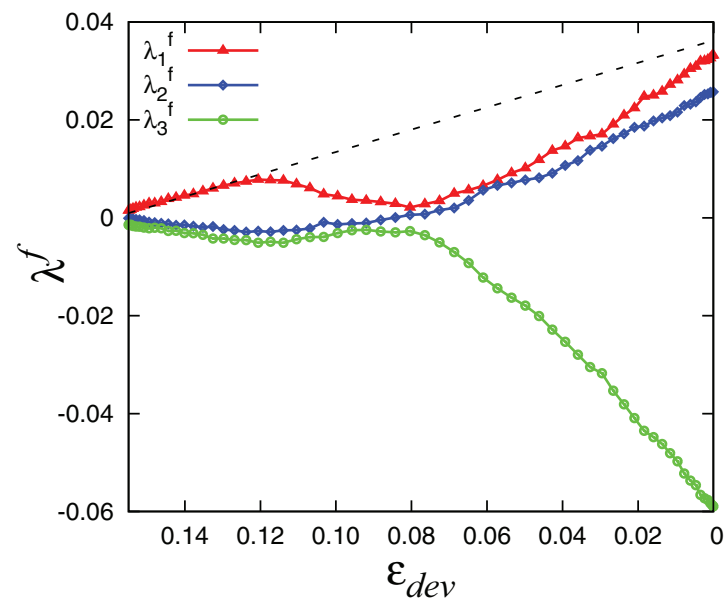

(b)

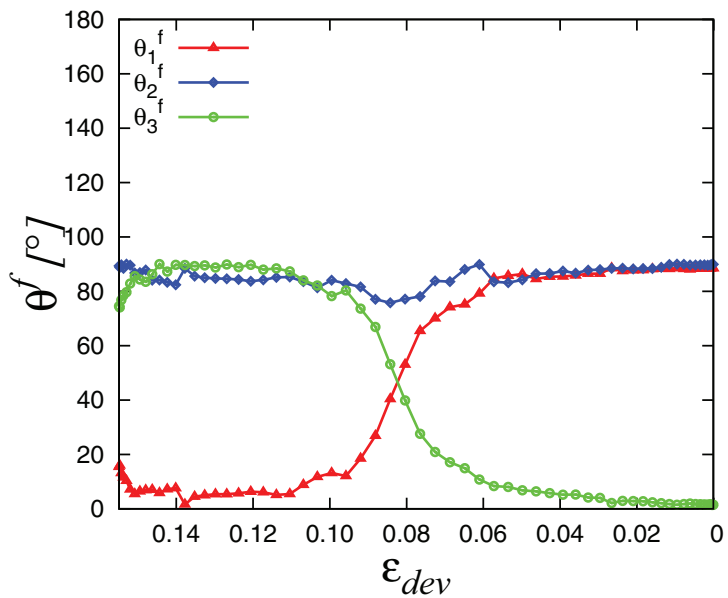

(d)

FIG. 6. (Color online) Eigenvalues of the deviatoric fabric for $\mu=0.1$ plotted as functions of the deviatoric strain for (a) loading and (b) unloading along with their corresponding orientations with respect to the compressive direction during uniaxial (c) loading and (d) unloading. Dotted lines indicated the slope of the path, identical before and after strain reversal.

friction possibly due to the higher maximum deviatoric stress values reported with increasing friction. The corresponding orientation of the major eigenvector of fabric $\theta_{1(f)}$ for all contacts and different friction coefficients also starts from different random values before decreasing to $0^{\circ}$ with increasing loading. Surprisingly at $\varepsilon_{\mathrm{dev}}=0.08$, for the configurations with lower friction $(\mu=0,0.01,0.02$, and 0.05$), \theta_{1(f)}$ remains close to $0^{\circ}$ while those with higher friction $(\mu=0.2,0.3,0.5$, and 1.0) begin to increase towards $90^{\circ}$ as we approach maximum compression. This indicates that the orientations and build-up of contacts for systems with lower or higher friction behave in an opposite fashion to each other and makes clear the reason for

TABLE II. Shape factors of deviatoric stress and deviatoric fabric in the respective tensor eigensystem at the beginning, maximum, and end of uniaxial compression.

\begin{tabular}{lllc}
\hline \hline Shape factor & $\tau \approx 0$ & $\tau \approx 0.5$ & $\tau \approx 1$ \\
\hline$\Lambda^{\sigma}=\lambda_{2}^{s} / \lambda_{1}^{s}$ & random & $-1 / 2$ & 1 \\
$\Lambda^{f}=\lambda_{2}^{f} / \lambda_{1}^{f}$ & random & $-1 / 2$ & 1 \\
$\Lambda^{(-\epsilon)}=\epsilon_{d}^{(2)} / \epsilon_{d}^{(1)}$ & undefined & $-1 / 2$ & 1 \\
\hline \hline
\end{tabular}

the decrease seen in the deviatoric fabric evolution in Fig. 6(a). At the beginning and with increasing loading, contacts are mostly built along the main compression direction. However with increasing friction, a "saturation" of contact build-up in the vertical direction sets in and an increasing number of contacts begin to build-up in the horizontal direction. As strain is reversed, the eigenvector orientation for systems with low friction increases to $90^{\circ}$ while a decrease before an increase to $90^{\circ}$ is seen for systems with higher friction.

We also plot the respective shape factors as a ratio of the eigenvalues of stress and fabric for some exemplary friction coefficients during uniaxial loading and unloading in Fig. 7. For stress, shown in Fig. 7(a), beginning from random values, $\Lambda^{\sigma}$ decreases to $-1 / 2$ during loading and reverses to 1 at the end of the unloading cycle. The rates of change during loading and unloading are almost identical, for different $\mu$ while during unloading, the deviatoric strain at which the increase occurs decreases with increasing friction. As with the stress, the shape factor of fabric $\Lambda^{f}$, shown in Fig. 7(b), also begins from random values and during loading approaches $\Lambda^{f} \approx-1 / 2$ with stronger fluctuations for higher friction coefficients. At the end of unloading however $\Lambda^{f}$ approach unity. 

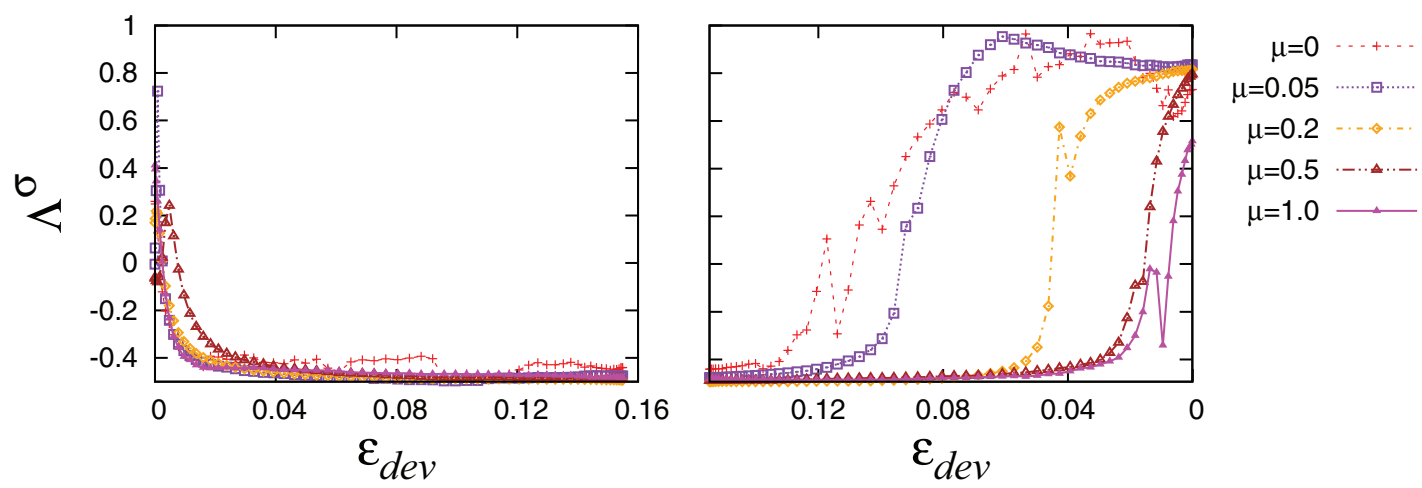

(a)
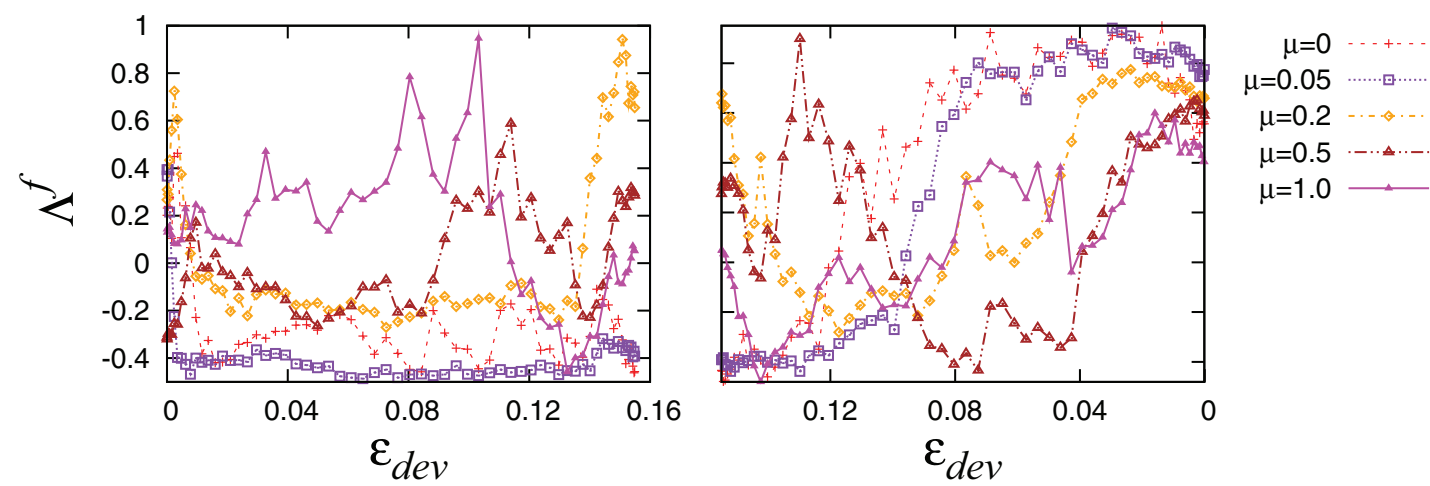

(b)

FIG. 7. (Color online) Shape factors of (a) stress and (b) fabric as function of the deviatoric strain for some exemplary friction coefficients.

\section{Weak and strong subnetworks}

To further understand this interesting observation we subdivide the respective systems into strong and weak contacts and we plot the orientation of the stress and fabric eigenvector corresponding to the compression direction for the two subdivisions. Strong contacts are termed as those whose normal force intensity is greater than the mean normal force while those with lower intensity with respect to the mean normal force are termed weak.

We plot the orientation of the major direction eigenvector of stress and fabric respectively in Figs. 8(c) and 8(d) for strong contacts. From Fig. 8(c), the orientation of the strong contact main eigenvector of stress and fabric behaves in a similar fashion as the total contact in the ensemble. This is consistent with earlier findings [16] where the strong contacts have been observed to carry most of the load during deformation. Interestingly and in contrast to the observation for all contacts, the fabric eigenvalue for systems with both low and high friction all stay close to $0^{\circ}$ during loading and initial unloading.

Next, the orientation of the main eigenvector of stress and fabric for weak contacts is shown in Figs. 8(e) and 8(f). Similar to the strong contacts, the stress and fabric orientation of weak contacts are mostly oriented at $90^{\circ}$ during loading. During unloading, the orientation tends towards $0^{\circ}$.

Comparing Figs. 8(b), 8(d), and 8(f), it can be seen that strong contacts predominate for the system with very low friction while for higher friction, the orientation of the weak contacts plays a much more significant role.
In Fig. 9, we plot the deviatoric strains at which the major eigenvalues $\theta_{1}^{s}$ cross $45^{\circ}$ during unloading for different friction coefficients. Additionally, we also plot the deviatoric strains at which the deviatoric stress ratio, deviatoric fabric, and the stress shape factor cross zero from Figs. 3(b), 3(d), and 7(a), respectively. As shown, the transition point decreases nonlinearly with increasing friction. All data originating from the stress tensor, namely the major eigenvalue of stress, its orientation, and the stress shape factor, all collapse on each other. On the other hand, it is not surprising that the transition points for the fabric quantities are slightly off since the fabric behaves differently from the stress. The definition of the fabric tensor takes into account only the normal directions and does not include the strong tangential contributions to the force introduced by friction. Therefore, as friction is increased, the deviations can be stronger.

In the following section, we will investigate in more detail the fraction of weak and strong contacts in these systems and discuss their interplay and relation to the observations on the orientations of the strong and weak contacts. For clarity and to better view the evolution of the quantities, instead of the deviatoric strain $\epsilon_{\mathrm{dev}}$, we will study the evolution of the quantities against dimensionless time $\tau=t / T$, where $T$ is the simulation time.

\section{E. Friction mobilization}

Mobilization of contact friction, during uniaxial deformation of the bulk material, is quantified by the factor $f_{t} / \mu f_{n} \leqslant 1$ 


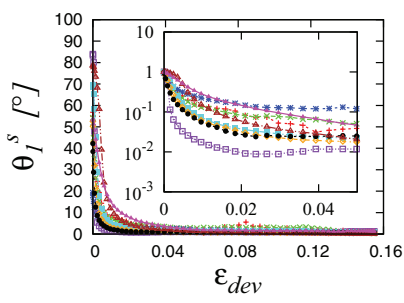

(a)
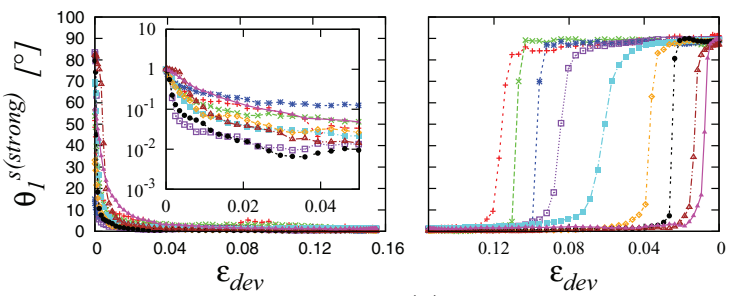

(c)
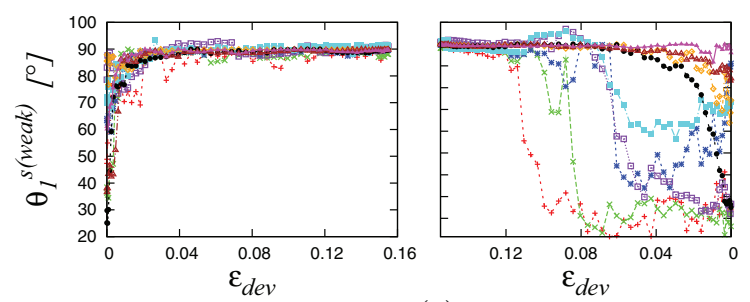

(e)

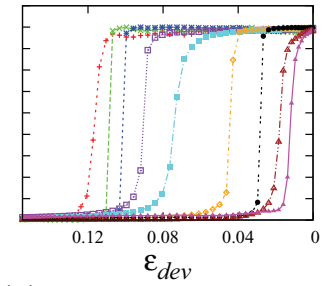

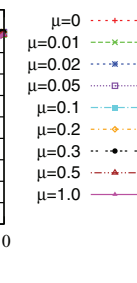
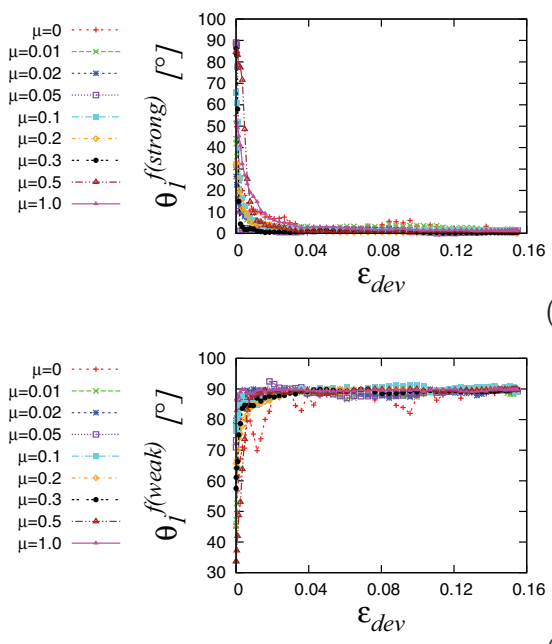

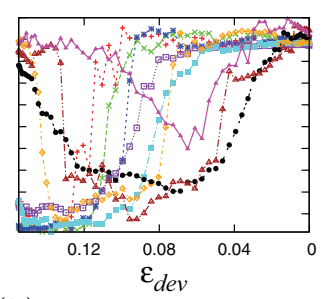

(b)

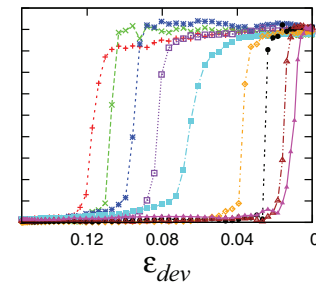

(d)

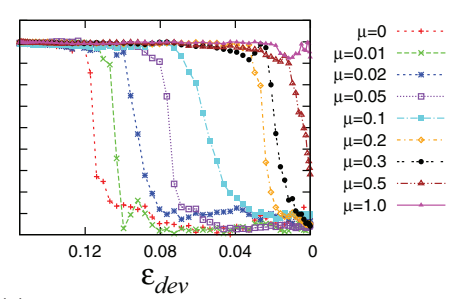

(f)

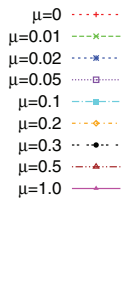

$\mu=0 \cdots+\cdots$ $\mu=0.01 \cdots$

$\mu=0.05$ $\mu=0.2 \ldots$ $\mu=0.3 \cdots \cdots$ $\mu=0.5 \cdots$ $\mu=1.0$

FIG. 8. (Color online) Orientation of the largest positive (a) stress eigenvector for all contacts, (b) fabric eigenvector for all contacts, (c) stress eigenvector for strong contacts, (d) fabric eigenvector for strong contacts, (e) stress eigenvector for weak contacts, (f) fabric eigenvector for weak contacts plotted against dimensionless time for different coefficient of friction.

for each contact. The tangential forces grow towards their limit and support larger shear stress; for tangential forces at or above the Coulomb limit, i.e., at fully mobilized friction, sliding sets in and rearrangements of contacts can lead to new, more stable configurations. It has been observed [50] that sliding is mostly active at weak contacts (termed weak sliding, $w s l$ ), while stronger contacts stay in the sticking regime and sustain

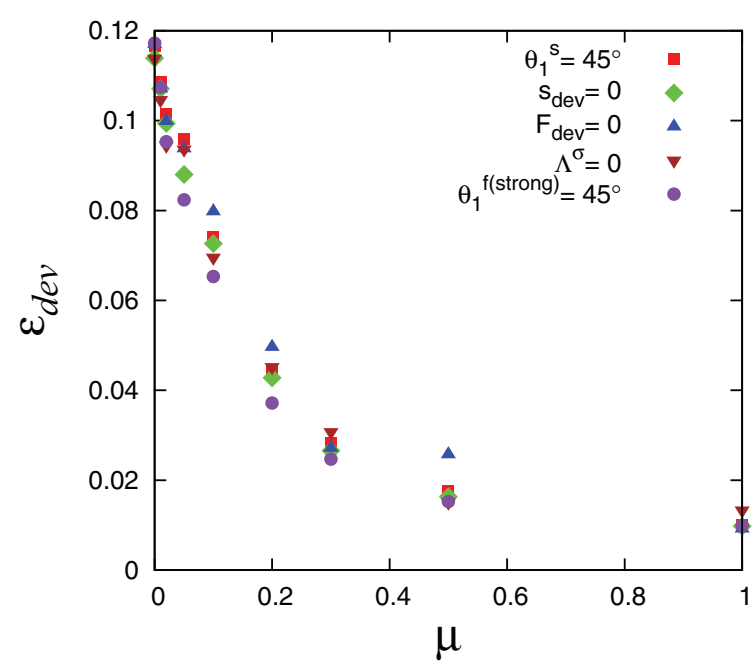

FIG. 9. (Color online) Strains at which the orientations of the stress eigenvectors cross $\theta=45^{\circ}$ and at which the deviatoric stress ratio, deviatoric fabric, and the stress shape factor cross zero for frictions $\mu=0,0.01,0.02,0.05,0.1,0.2,0.5$, and 1.0 . larger friction forces while being less mobilized (termed strong sticking $s s t$ ). We refer to this as the $w s$ rule. Weak and strong contacts are defined relative to the average normal force at each time step;

$$
f^{*}=f_{n} /\left\langle f_{n}\right\rangle<1
$$

are termed weak and

$$
f^{*}=f_{n} /\left\langle f_{n}\right\rangle>1
$$

are termed strong [50], with dominating sliding and sticking, respectively.

As we will see shortly, we find that this friction mobilization rule may not strictly hold in certain cases, as there may be a considerable number of weak contacts with friction not fully mobilized (termed weak sticking, wst), as well as strong contacts fully mobilized (termed strong sliding, $s s t$ ).

As representative examples, in Fig. 10 we track two different contacting pairs during uniaxial loading and unloading of the system with $\mu=0.1$ and study the force intensity and friction mobilization as they evolve as function of the dimensionless time $\tau$. For the first contact pair shown in Fig. 10(a), during the first stages of loading, the contact is weak since $f^{*}<1$; friction is fully mobilized and sliding occurs at the contact, i.e., weak contacts tend to full friction mobilization. For a short period at $\tau \approx 0.2$, the contact becomes stronger and $f_{t} / \mu f_{n}$ correspondingly reduces (with strong fluctuations) indicating a strong contact where sticking predominates. At $\tau \approx 0.36$, the contact between this particle pair is lost (opened) and is only recovered at $\tau \approx 0.7$, where it 


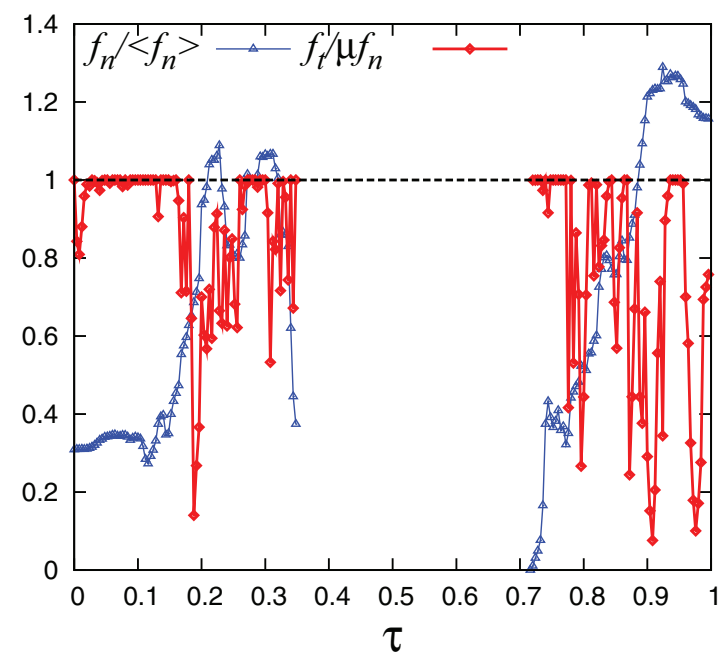

(a)

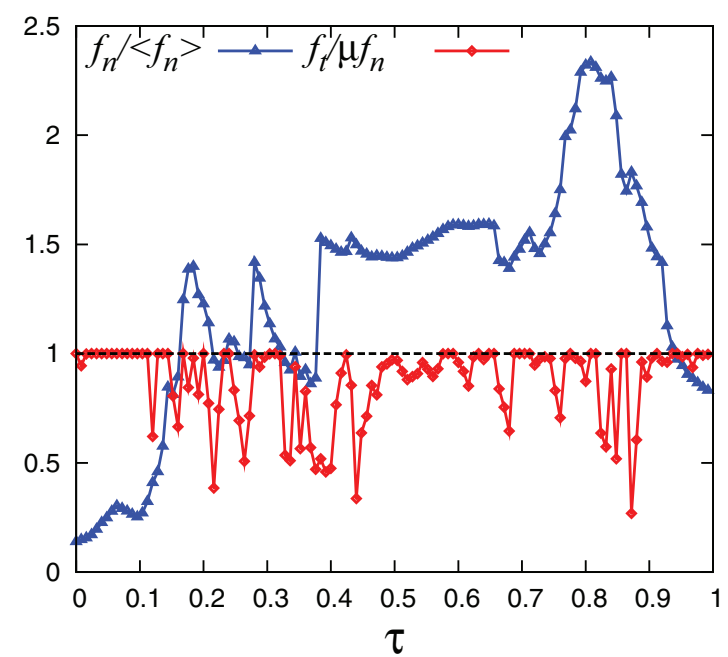

(b)

FIG. 10. (Color online) Tracking $f_{t} / \mu f_{n}$ and $f^{*}=f_{n} /\left\langle f_{n}\right\rangle$ for two single particle pairs randomly selected from the ensemble during compression and decompression where $\tau$ is the dimensionless time. (a) Particle pair 1. (b) Particle pair 2.

can again be classified as a weak sliding $(w s l)$ contact. As the end of the compression cycle is reached, the contact intensity increases and $f_{t} / \mu f_{n}$ decreases, with strong fluctuations again, and sometimes sliding. In general, the ws rule is mostly true for this contact pair except during the transition from weak to strong where some fluctuations in $f_{t} / \mu f_{n}$ can be seen; transitions from sliding to sticking can happen for weak contacts (wst) well below $f^{*}=1$ during increase of $f^{*}$, but also sliding can happen for strong contacts (ssl).

The second contact pair shown in Fig. 10(b) is even more interesting. Like the first particle pair, the second pair also begins as a weak sliding contact and $f^{*}$ grows until $\tau \approx 0.15$, where it becomes strong. Interestingly, while the contact remains very strong for almost all of the loading-unloading cycle, friction is highly mobilized; $f_{t} / \mu f_{n}$ remains close to 1 .

Since studying just two contact pairs within an ensemble containing tens of thousands of contacts provides very little information, we first extract the total fraction of weak and strong contacts in the system. In Fig. 11(a), we plot the total proportion of weak contacts with reference to the total number of contacts for the different friction coefficients (which was studied in detail in Refs. [20,42], so those data are not shown here). Surprisingly, as with the orientation of the largest eigenvalue of fabric for weak and strong forces plotted in Fig. 8, we see a clear difference between the fraction of weak and strong contacts. In the following, we will discuss in detail the observations for weak contacts-which have opposite trends as the observations for strong contacts.

The first observation from Fig. 11(a) is that a greater fraction (over $50 \%$ ) of the contacts in the respective systems are weak-an indication that fewer contacts carry a larger proportion of the load in the system, which is related to the shape of the force probability density function $P\left(f^{*}\right)$; see Sec. IV F. Second, for systems with lower friction, the fraction of weak contacts at the beginning of the loading cycle is significantly higher than for higher friction, meaning that the load is more evenly (not exactly proportionally) distributed between weak and strong contacts for systems with higher

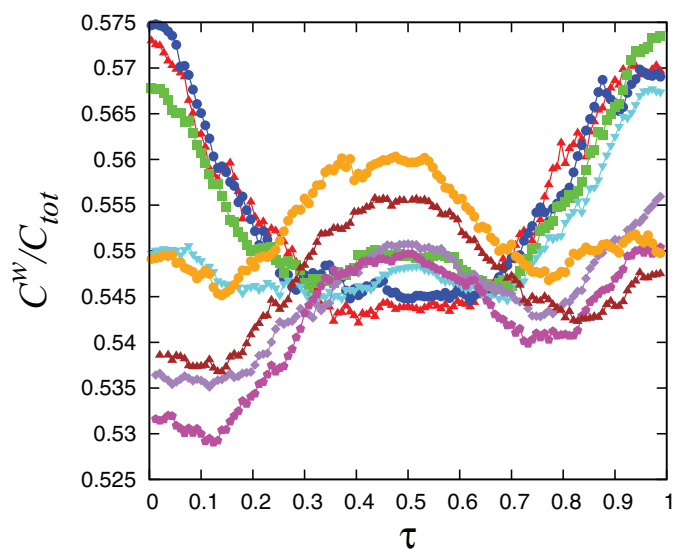

(a)

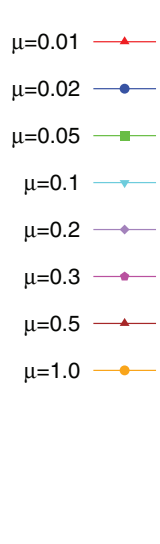

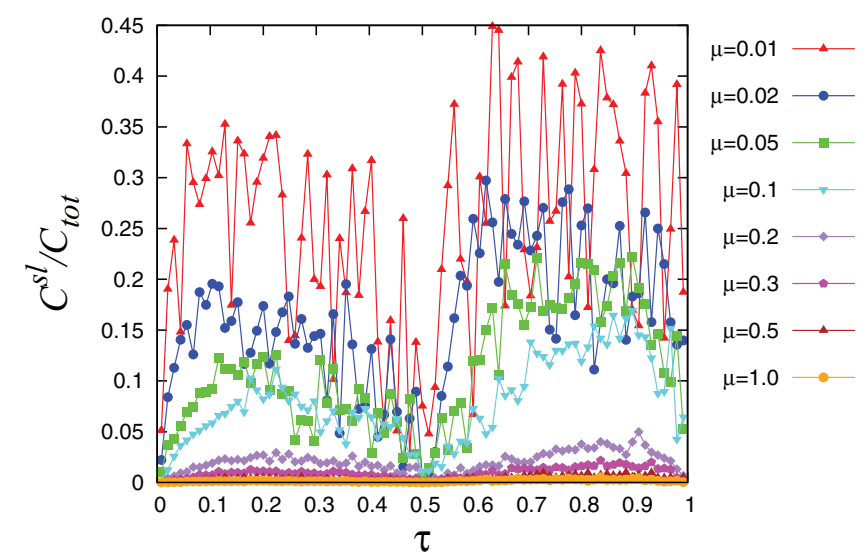

(b)

FIG. 11. (Color online) Proportion of (a) weak contacts (b) sliding contacts with respect to the total number of contacts during uniaxial loading and unloading cycle for different friction coefficients. 
friction coefficient. With increasing loading, while the total number $C_{\text {tot }}$ strongly increases [see Fig. 2(c)], the fraction of weak contacts decreases for packings with lower friction coefficients, and increases for those with higher friction. Also, the decrease of weak contacts with increasing loading for lower friction systems is stronger and occurs earlier than the increase for systems with higher friction. At maximum loading $\tau=0.5$, the proportion of weak contacts are close for all friction coefficients with slightly higher fraction for the highest friction coefficients $\mu=0.5$ and 1.0. This observation, that the packings with higher friction behave in a qualitatively different fashion, is consistent with the earlier observation in Fig. 8(b), where the difference in orientation of strong or weak contacts for low or high friction coefficients can be seen too.

It is surprising that the fractions of weak contacts are close for systems with lower friction and evolve in a similar (almost symmetric) fashion during loading and unloading. For $\mu=$ 0.01 and 0.05 , the fractions of weak contacts at the end of unloading are slightly lower than at the beginning of loading. With increasing friction, the fractions of weak contacts at the end of unloading are higher than at the beginning of loading; the antisymmetry between the loading and unloading phases is more visible for $\mu \geqslant 0.1$.

From Fig. 11(b), we plot the number of sliding contacts with respect to the total contacts for different contact friction coefficients. The number of sliding contacts increases as the contact friction coefficient reduces with stronger fluctuations for lower contact friction. The configurations with low friction are less stable and require more contacts to stabilize them. This contrasts with the observation when contact friction is high, where fewer contacts are needed for stability. Also, the fluctuations for lower friction coefficient resemble stick-slip behavior where sliding and sticking alternate. Comparing the kinetic energies for low and high friction, we observe that the kinetic energy reduces with increasing friction leading to higher kinetic energy during slip events and thus stronger fluctuations for the systems with lower friction coefficients.

To evaluate the proportion of weak and strong contacts contributing to sliding and sticking at contacts, we plot in Fig. 12, the number of weak sliding $\left(\sum w s l\right)$ and strong sticking $\left(\sum s s t\right)$ contacts with respect to the the total weak $\left(\sum w\right)$

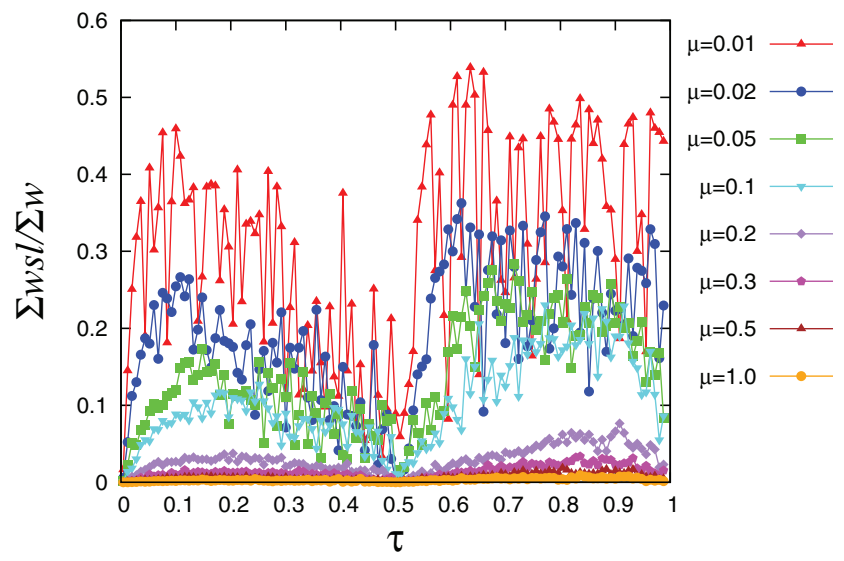

(a) and strong $\left(\sum s\right)$ contacts, respectively. From Fig. 12(a), the fraction of weak sliding contacts grows during loading and reaches a peak before it begins to decrease towards zero as maximum loading $(\tau=0.5)$ is approached. The initial growth rate of the weak sliding contacts and the peak reached decreases with increasing friction but all approach zero at $\tau=0.5$ because the deformation rate decreases to zero before reversal. During unloading, a second growth phase of the weak sliding contacts is seen and the maximum reached is higher than that reached during loading-thus leading to nonsymmetry around $\tau=0.5$. Additionally, only a small proportion (much less than $\approx 50 \%$ ) of the total weak contacts are sliding. This indicates that even though an increase in the number of weak sliding contacts is seen during loading and unloading, more and more weak contacts stick $\left(f_{t} / \mu f_{n}<1\right)$ for increasing $\mu$.

In contrast to the weak sliding contacts, the fraction of strong sticking contacts, as presented in Fig. 12(b), decreases during loading until it reaches a minimum before an increase towards $\tau=0.5$ can be seen. The rate of decrease and the minima reached decrease with increasing friction and the minima are lower during unloading, i.e., all data are nonsymmetric around $\tau=0.5$.

In Fig. 13, we plot the number of weak sliding $\left(\sum w s l\right)$ and strong sticking $\left(\sum s s t\right)$ contacts with respect to the total sliding and sticking contacts, respectively. In Fig. 13(a), we confirm that a higher proportion $(>0.5)$ of the sliding contacts are weak [50-53]. The proportions of weak sliding contacts for $\mu=0.01-0.3$ are almost identical and decrease during loading. During unloading, however, the proportions of weak sliding contacts behave differently with increasing friction. We again observe the nonsymmetry of the loading and unloading data. In Fig. 13(b), we plot the fraction of strong sticking contacts with respect to the total sticking contacts. A little less than $50 \%$ of the sticking contacts are strong. The fractions of strong sticking contacts increase initially during loading and later decrease as maximum compression is approached. The fraction of strong sticking contacts show a decreasing trend at $\tau=0.5$ with increasing $\mu$. During unloading, the fractions of strong sticking contacts increase and later decrease towards the end of the unloading branch. With increasing friction, the

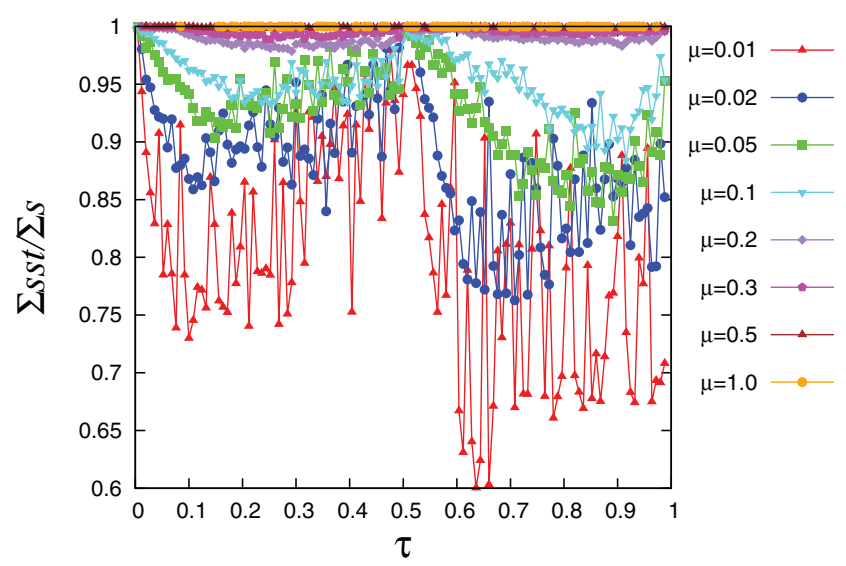

(b)

FIG. 12. (Color online) Fraction of (a) weak sliding contacts $(w s l)$ and (b) strong sticking contacts (sst) with respect to the total number of weak $\left(\sum w\right)$ and strong $\left(\sum s\right)$ contacts, respectively, during uniaxial loading and unloading for different friction coefficients. 


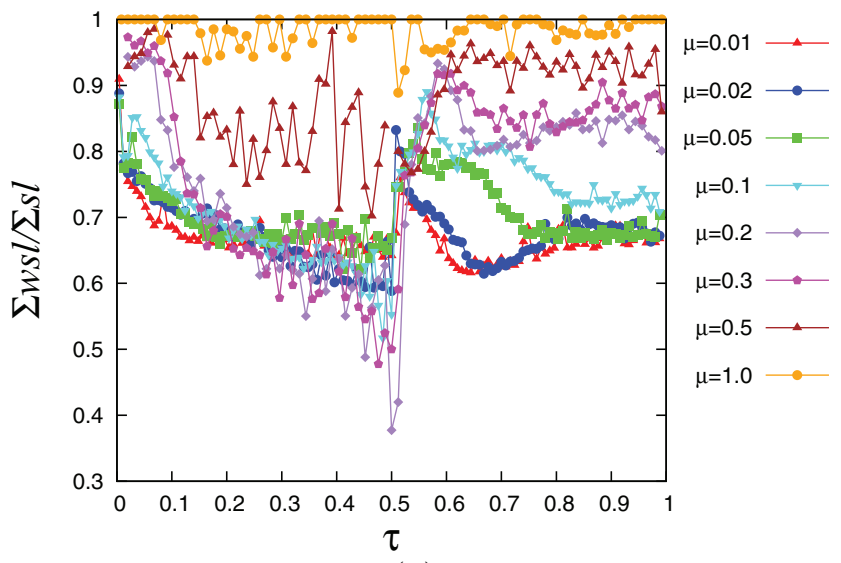

(a)

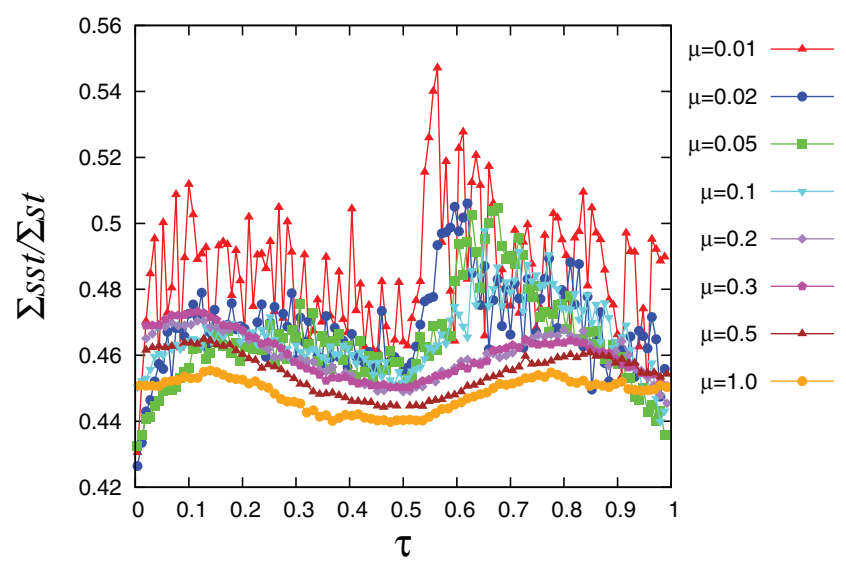

(b)

FIG. 13. (Color online) Fraction of (a) weak sliding contacts $(w s l)$ and (b) strong sticking contacts (sst) with respect to the total number of sliding $\left(\sum s l\right)$ and sticking $\left(\sum s t\right)$ contacts, respectively, during uniaxial loading and unloading for different friction coefficients.

nonsymmetry of the data decreases. For the highest friction coefficients, the fraction of strong sticking contacts during loading is slightly more than those present during unloading.

In summary, strong and weak forces have been analyzed along with the level of friction mobilization. It has been shown that a higher proportion of the total contacts in the system are weak, irrespective of the friction coefficient. Among these weak contacts, the contacts which are sliding are less in number compared to the sticking contacts. In contrast, when the total sliding contacts are considered, a higher proportion of them are weak, as also reported in earlier literature.

\section{F. Probability density function}

To better understand the relationship between contact forces and the macroscopic stress and structure, we study the probability density function of normal contact forces in different directions [48,54]. We will consider the probability distribution of the normal forces during uniaxial compression with reference to the compressive $z$ direction and the two lateral $x$ and $y$ direction. Keeping each direction as reference, we define a cutoff $\chi$ such that the contact forces admitted for the probability distribution analysis fulfill the criteria $\left|\hat{n}_{c} \cdot \hat{n}_{\epsilon}\right|>\chi$ where $\hat{n}_{c}$ is the normal unit vector of the reference direction and $\hat{n}_{\epsilon}$ is the strain eigenvector corresponding to a compressive or tensile direction. The strain eigenvector is fixed due to the deformation mode, but will be different for other test setups. In the case $\chi=0$, all contact forces in the ensemble will be considered while no contacts exist when $\chi=1$. For the present study, we set $\chi=0.8$ and only note that as $\chi$ approaches 1 , less data are available and the noise level increases, but the general trend of the results is not affected.

In Fig. 14, we plot the normalized probability density of the normal force $P\left(f /\left\langle f_{\text {all }}\right\rangle\right)$ against the normalized force $f /\left\langle f_{\text {all }}\right\rangle$ for the three reference directions $(x, y$, and $z)$ and for all contacts. In this case, $\mu=0.1$ and cutoff $\chi$ has been set to 0.8 . To allow for comparison, the forces have been normalized with the mean of the normal force for all contacts. As shown in Fig. 14(a), at $\tau=0$, the force probabilities from the three reference directions and for all contacts are virtually the same evidenced by the apparent collapse of the different curves on each other. This is not surprising since the initial state is isotropic and no direction-dependent deformation has taken place. At maximum compression [in Fig. 14(b)], a difference between the force distribution in the compression $z$ direction and the radial $x$ and $y$ direction is evident. First, we

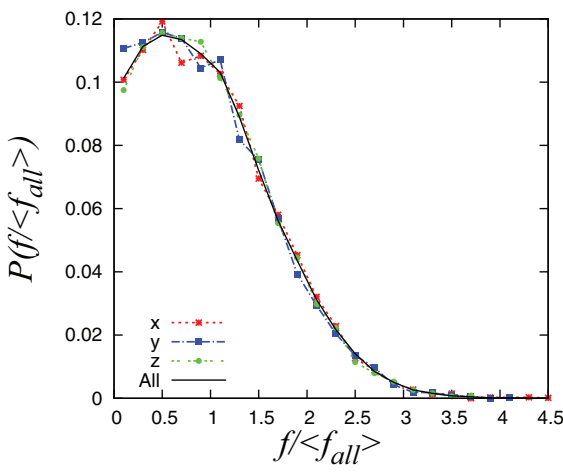

(a)

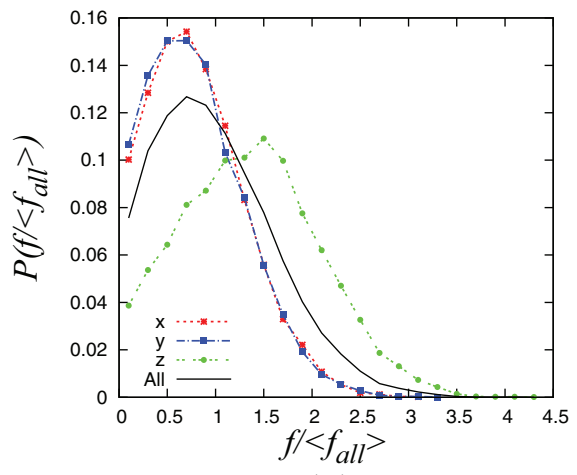

(b)

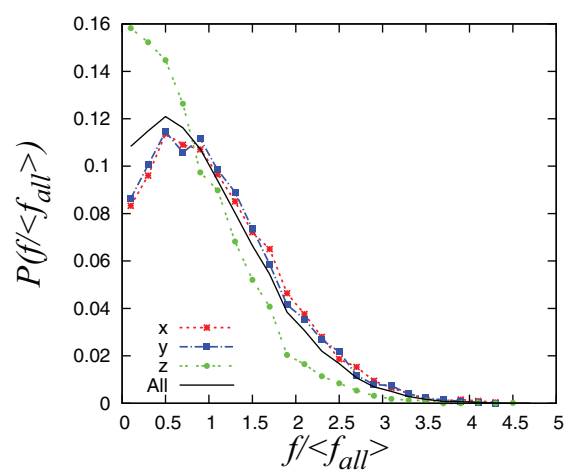

(c)

FIG. 14. (Color online) Normalized probability density of the normal force $P\left(f /\left\langle f_{\text {all }}\right\rangle\right)$ for the three reference directions and for all contact forces plotted against the normalized force $f_{*}=f /\left\langle f_{\text {all }}\right\rangle$ for $\mu=0.1$ and cutoff $\chi=0.8$. Three snapshots are shown at (a) initial state $\tau=0$, (b) maximum, $\tau=0.5$, and (c) final state $\tau=1.0$. 
observe that the force probability in the radial directions $(x, y)$ are close since no relative deformation takes place in these directions. Another observation is that the proportion of weak forces in the radial direction significantly exceeds those in the compression direction. Also, the mean force in the $z$ direction is observed to be higher than in the radial direction. Finally, at maximum compression, we observe a wider distribution in the compressive $z$ direction compared to the force distribution for all contacts and the two lateral directions. The longer tail seen in the force distribution in the $z$ direction is due to the presence of stronger forces compared to the other directions.

At the end of the decompression cycle, shown in Fig. 14(c), we observe that the initial state is not recovered due to the deformation history of the sample and there is a higher proportion of weak forces in the decompression $z$ direction compared to the radial directions.

\section{POLAR REPRESENTATION}

To understand the orientation and arrangement of the contacts over the whole angular spectrum during uniaxial deformations, we introduce now the polar representation of contacts, forces, and mobilized friction. For the analysis, we test two different averaging methods, namely the constant bin width $(b)$ and constant height $(h)$, which give comparable results and are shown in detail in Appendix A. In the following, we will use data obtained using the constant bin width method.

\section{A. Harmonic approximations}

The axial distribution of contact force orientations $P(\theta)$, along with the degree of anisotropy in a granular packing, can be approximated by a Legendre polynomial based on spherical harmonics of the form $Y_{l}^{m}(\theta, \varphi)$ [48,54-56]. The approximation is simplified by admitting only functions that are consistent with the symmetry of the deformation mode, namely functions independent with respect to $\varphi$ and periodic as a function of $\theta$. With these criteria, the two lowest admissible functions are $Y_{0}^{0}=1$ and $Y_{2}^{0}=3 \cos ^{2} \theta-1$ such that the second order harmonic representation of contacts is of the form

$$
P_{2}(\theta)=a_{0}\left[1+\epsilon\left(3 \cos ^{2} \theta-1\right)\right]
$$

with the factor $a_{0}$ as constant and a unique anisotropy descriptor $\epsilon$. In our case, due to normalization, $a_{0} \approx 0.5$. For the uniaxial mode, snapshots of the contact probability density data are presented in Fig. 16(a) during uniaxial loading and unloading. We observe distributions with two peaks and a dip around $\pi / 2$ indicating that a higher approximation with order higher than 2 (when only one peak is expected) is needed. The higher order required to describe the present uniaxial dataset is possibly due to the peculiarity of the deformation mode. Unlike the triaxial test which involves an active stress control on the lateral boundaries of the system, the stress on the lateral boundaries of the uniaxial mode evolve, albeit with smaller magnitude in comparison to the stress in the axial direction.

Equation (24) can be extended to admit higher order spherical harmonic functions with $l=4,6$. For $l=4, Y_{4}^{0}=$ $35 \cos ^{4} \theta-30 \cos ^{2} \theta+3$ and for $l=6, Y_{6}^{0}=231 \cos ^{6} \theta-$ $315 \cos ^{4} \theta+105 \cos ^{2} \theta-5$ all with different prefactors. For a sixth order expansion, the contact distribution will take the
TABLE III. Second and sixth order harmonic expansion of the contact distribution for the axial direction (compression: $\theta=0, \pi$ ) and the lateral direction $(\theta=\pi / 2)$.

\begin{tabular}{lcc}
\hline \hline$\theta$ & $P_{2}(\theta)$ & $P_{6}(\theta)$ \\
\hline$\theta=0$ & $a_{0}[1+2 \epsilon]$ & $a_{0}\left[1+2 \epsilon_{2}+8 \epsilon_{4}+16 \epsilon_{6}\right]$ \\
$\theta=\pi / 2$ & $a_{0}[1-\epsilon]$ & $a_{0}\left[1-\epsilon_{2}+3 \epsilon_{4}-5 \epsilon_{6}\right]$ \\
$\theta=\pi$ & $a_{0}[1+2 \epsilon]$ & $a_{0}\left[1+2 \epsilon_{2}+8 \epsilon_{4}+16 \epsilon_{6}\right]$ \\
\hline \hline
\end{tabular}

form

$$
P_{6}(\theta)=a_{0}\left[1+\epsilon_{2} Y_{2}^{0}+\epsilon_{4} Y_{4}^{0}+\epsilon_{6} Y_{6}^{0}\right],
$$

where the axial symmetry is implied. Equation (25) introduces now three anisotropy state descriptors $\epsilon_{i}$, with $i=2,4,6$. Also, Eqs. (24) and (25) can be further simplified for the well defined limits at $\theta=0, \pi / 2$ and $\pi$ as shown in Table III.

Different methods of obtaining the anisotropy state descriptors have been attempted in this study. The details and comparison of the methods are discussed in Appendix B. For all methods, we consistently observe that the contact distribution is approximated by a sixth order polynomial with two peaks and a strong depression at $\pi / 2$. In the following, as a reference case, we use the azimuthal fit to the constant probability data discussed in Appendix B.

\section{B. Discussion of results}

Having established that the contact distribution is approximated by a sixth order distribution with three anisotropy state descriptors, we compare descriptors $\epsilon_{2}, \epsilon_{4}$, and $\epsilon_{6}$ for different friction coefficients as functions of the deviatoric strain during uniaxial loading and unloading. From Fig. 15(a), besides a slight increase in the maximum $\epsilon_{2}$ values between $\mu=0$ and 0.02 , the maximum $\epsilon_{2}$ value shows a decreasing trend with friction and almost saturates for the highest friction coefficients. This is consistent with the trend of the maximum deviatoric fabric shown in Fig. 4. Also consistent with the deviatoric fabric evolution during unloading is that the initial state is not recovered. In Fig. 15(b), beginning from different random values, $\epsilon_{4}$ is negative and systematically decreases for all friction coefficients during loading followed by a slight increase during unloading. The descriptor $\epsilon_{6}$ is distributed around zero and remains fairly constant during loading and unloading but has some variation within either deformation.

In addition to the contact probabilities in Fig. 16(a), we now study the probability distribution for other quantities. The polar distributions of the normal force shown in Fig. 16(b) during loading displays a high and increasing normal force along the compression $\left(0^{\circ}\right)$ direction compared to the lateral $(\pi / 2)$ direction reaching their maximum at $\tau=0.5$. After strain reversal (unloading), the normal force in the tensile (extension) direction is reduced until the force in the lateral $(\pi / 2)$ direction becomes higher. Interestingly, in contrast to $P(\theta)$, the distribution of the normal forces $f_{n}(\theta)$ is well described by a second-order harmonic approximation similar to Eq. (24) during loading and unloading.

For the distribution of the tangential force and mobilized friction, shown respectively in Figs. 16(c) and 16(d), we observe a distribution similar to that of the contacts shown in 

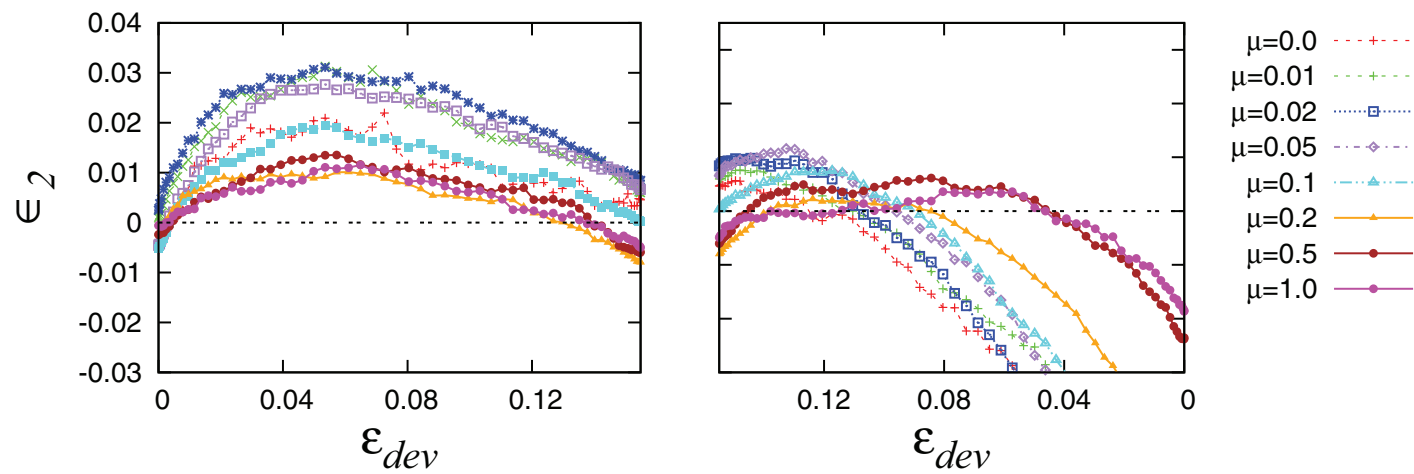

(a)
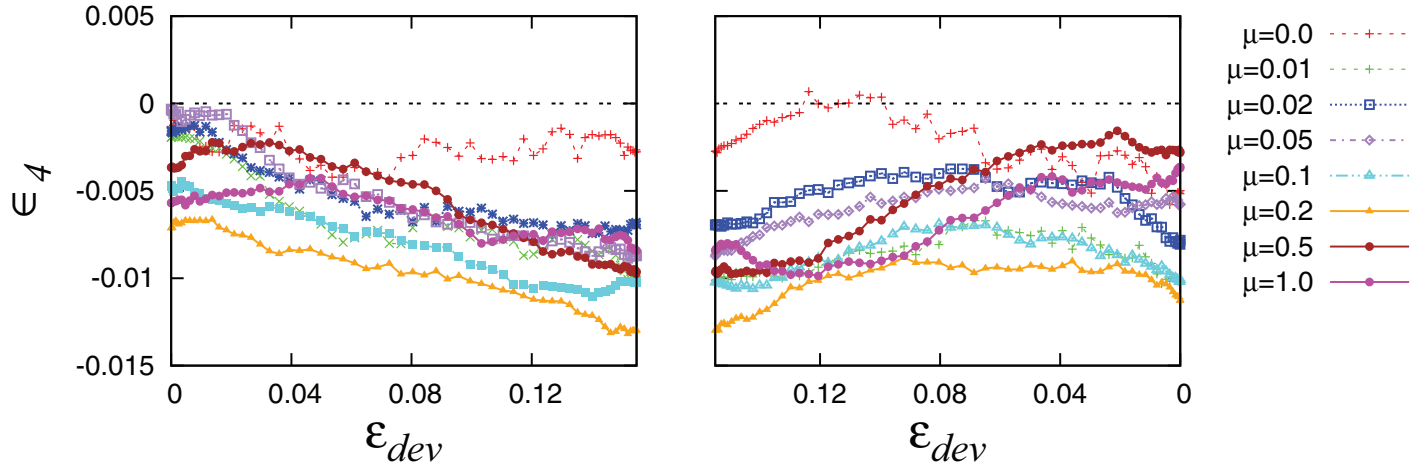

(b)
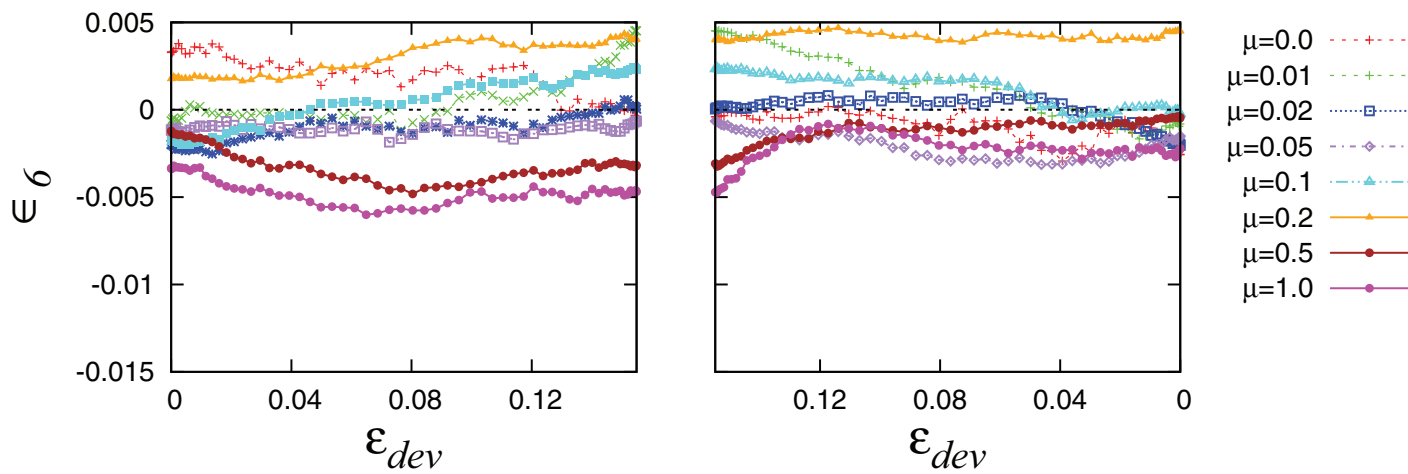

(c)

FIG. 15. (Color online) Comparison of the sixth order anisotropy state descriptors (a) $\epsilon_{2}$, (b) $\epsilon_{4}$, (c) $\epsilon_{6}$ as a function of the deviatoric strain for different friction coefficients during loading (left) and unloading (right).

Fig. 16(a), with two strong peaks and a depression around $\pi / 2$. This indicates the need for a higher order tensorial descriptor also for these two quantities that appear to be strongly related with the behavior of the contact network, rather than with the normal forces. Similar to $f_{n}(\theta)$, the distribution of the tangential force $f_{t}(\theta)$ also shows an increase along the compression direction followed by a decrease during decompression. We also find that during loading, the mobilized friction increases along the tensile $(\pi / 2)$ direction while remaining fairly stable and flat in the lateral direction. After strain reversal, the mobilized friction increases again along the tensile direction (which is now $0^{\circ}$ ). Coupling these observations to the normal force distribution, we find that friction is less mobilized along the direction where stronger forces exist (compression) and more mobilized along the direction where weaker forces (tension) are seen. Similar to the directional probability distribution of the normal force presented in Sec. IVF, the initial state (at $\tau=0$ ) which is mostly isotropic is not recovered at the end of unloading $(\tau=1)$. As a complement, in Fig. 17, we show the pictorial representation of the distributions of contacts, normal force, tangential force, and mobilized friction at $\tau=0.5$ for the same dataset shown in Fig. 16, clearly visualizing the $\epsilon_{4}$ and $\epsilon_{6}$ contributions in Figs. 17(a), 17(c), and 17(d).

\section{SUMMARY AND OUTLOOK}

The discrete element method has been used to investigate the microscopic and macroscopic response of frictional, polydisperse granular assemblies under uniaxial loading and unloading paths. The main goal was to investigate the effects of contact friction on the force and contact network orientation 


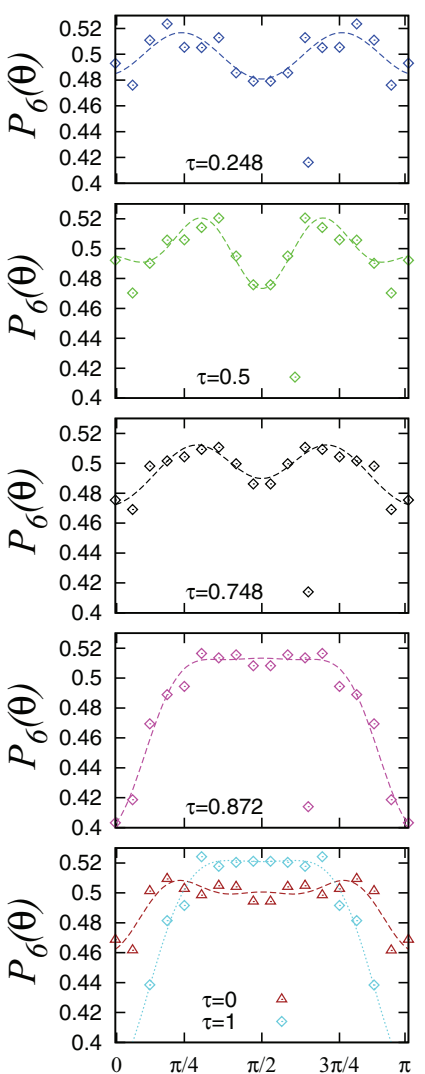

(a)

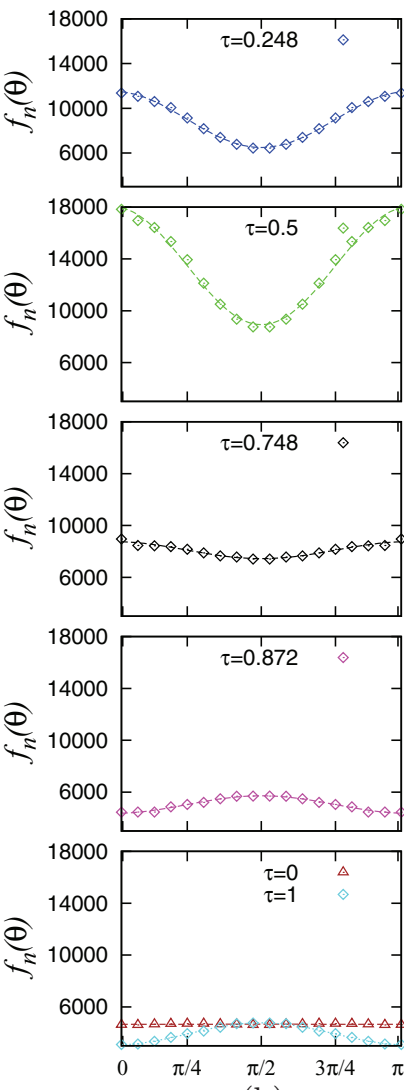

(b)

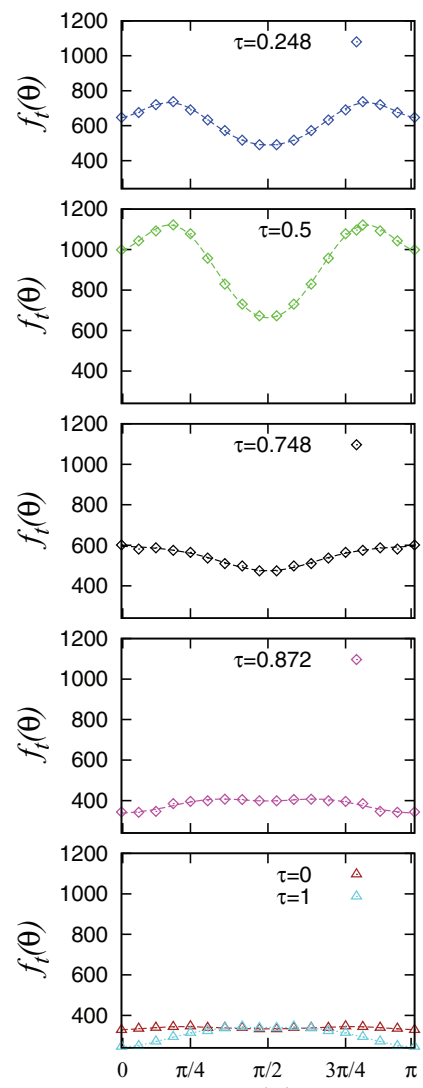

(c)

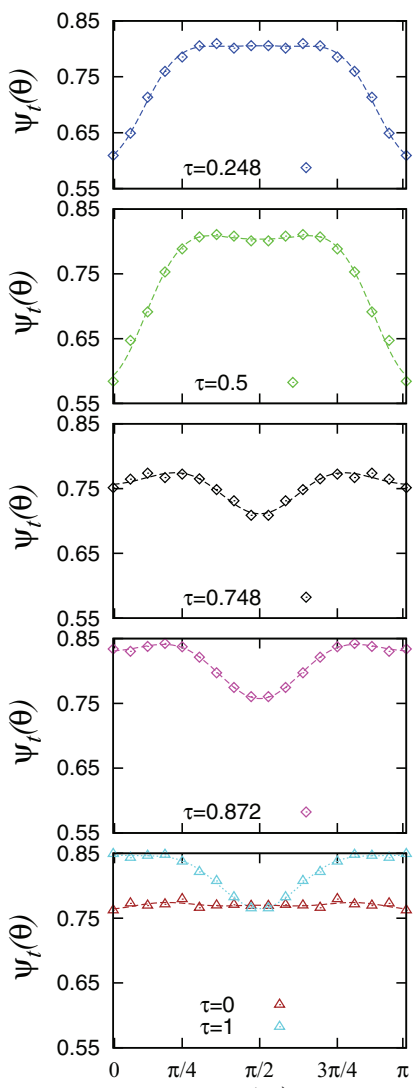

(d)

FIG. 16. (Color online) Snapshots of the polar distribution of the (a) contacts $P_{6}(\theta)$, (b) normal force $f_{n}(\theta)$, (c) tangential force $f_{t}(\theta)$, (d) mobilized friction $\psi_{t}(\theta)$ at different dimensionless time $(\tau)$ during uniaxial loading and unloading for friction $\mu=0.1$.

and distribution and to relate this to the evolution of structural anisotropy - which is the key ingredient that quantifies the response of granular materials under nonisotropic loading conditions. Since the uniaxial test is readily realizable in laboratory experiments, our findings should be relevant for both experimental and numerical research on the behavior of packings under different deformation and stress conditions. The present study covers a wide range of friction coefficients for systems that are already "jammed." Since the boundary walls are periodic, the effects of walls and system geometry should be minimal, which allows us to understand the bulk behavior with rather few particles $(N \approx 10000)$.

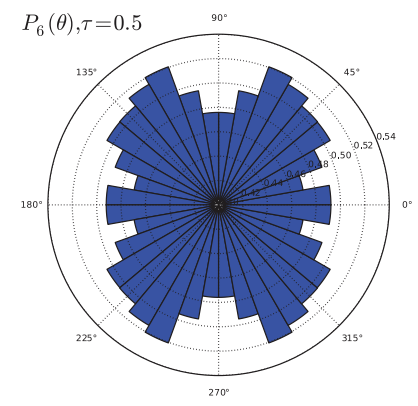

(a)

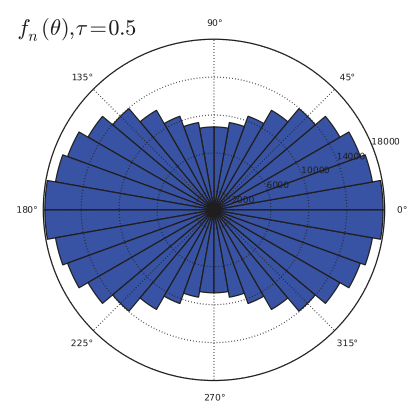

(b)

As preparation procedure, in order to obtain homogeneous initial isotropic states, we attempted several preparation protocols and found that the methods lead to mostly identical initial states even though care has to been taken in the presence of friction which leads to protocol dependence.

Given the same initial density, packings with different coefficients of friction are highly different in many respects. The evolution of the deviatoric stress ratio and the deviatoric fabric, as functions of the deviatoric strain, in the presence of friction, are different with respect to each other. Even though the contact model is linear, both quantities show a nonlinear behavior due to the structural changes during

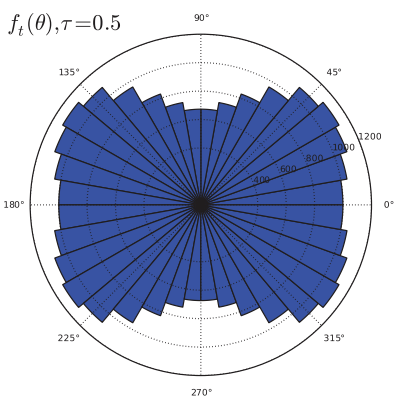

(c)

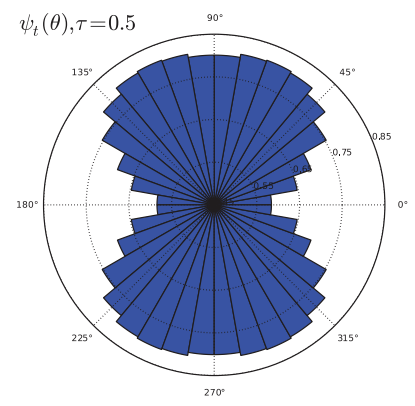

(d)

FIG. 17. (Color online) Polar distribution of the (a) contacts $P_{6}(\theta)$, (b) normal force $f_{n}(\theta)$, (c) tangential force $f_{t}(\theta)$, (d) mobilized friction $\psi_{t}(\theta)$ at dimensionless time $(\tau=0.5)$ for friction $\mu=0.1$. 
loading and unloading. For the configurations with lower friction, a saturation in the deviatoric stress ratio during loading was observed. However, when friction is increased, a clear saturation of the deviatoric stress ratio is not seen within the same maximum strain. During loading, the deviatoric fabric, which quantifies the structural anisotropy, reaches a maximum before maximum $\varepsilon_{\mathrm{dev}}$ independent of $\mu$, and then decreases as the maximum strain is approached. Interestingly for the higher friction coefficients, a second increase was observed. The peak deviatoric stress ratio $s_{\mathrm{dev}}^{\max }$ reached during uniaxial loading increases up to a peak at $s_{\mathrm{dev}}^{\max }=0.42$ and subsequently decreases for higher friction to $s_{\text {dev }}^{\max } \approx 0.33$. The peak deviatoric fabric reached, $F_{\mathrm{dev}}^{\max }$, largely shows a decreasing trend with increasing friction and eventually saturates at $F_{\mathrm{dev}}^{\max } \approx 0.025$.

The orientation of the largest stress eigenvector $\theta_{1}^{s}$, during loading, with nonsystematic rates, aligns to $0^{\circ}$, i.e., the compression direction. When strain is reversed, we observe that $\theta_{1}^{s}$ remains oriented along the vertical direction before reverting to $90^{\circ}$. The deviatoric strain at which the reversal happens is observed to decrease with increasing friction. On the other hand, the orientation of the largest fabric eigenvector $\theta_{1}^{f}$ shows a stronger dependence on friction. For systems with low friction, $\theta_{1}^{f}$ aligns with the compressive direction during loading, whereas the configurations with high friction begin to align perpendicular to the axial direction as maximum deviatoric strain is approached.

The deviatoric strains (relative to the original configuration) at which the stress tensor changes can be identified in different ways, i.e., $s_{\mathrm{dev}}=0, \theta_{1}^{s}=45^{\circ}$ and stress shape factor $\Lambda^{\sigma}=0$ (zero). All are identical to each other and show a decreasing dependence on friction, i.e., reversal happens later for larger $\mu$. Quantities relating to the microstructure, e.g., the strain at which, e.g., $F_{\mathrm{dev}}=0$, are different from the stress.

In comparison to other deformation protocols studied in literature, while the coordination number is found to decrease with increasing triaxial loading (or increasing deviatoric strain) $[8,57]$, we find that the coordination number always increases with strain under uniaxial compression. Under triaxial loading, the number of contacts is found to increase in the vertical (compression) direction while decreasing or dilating in the horizontal (fixed stress) direction [48], but in the uniaxial mode, the number of contacts is found to increase in both the horizontal and vertical directions. Also, the peak deviatoric fabric is found to increase with increasing friction under triaxial loading $[8,57]$, whereas in the uniaxial mode, the peak deviatoric fabric decreases with increasing friction.

As reported in previous studies [15], we also confirm that the orientations of the eigenvectors of stress corresponding to the compression direction for strong (forces greater than average) and weak (forces less than average) contacts are orthogonal with respect to each other. As a consequence of the definition of the stress tensor, the effects of strong contacts are more dominant for stress. For fabric, however, strong contacts are more dominant when friction is low while the weak contacts are found to play a bigger role for stronger friction, causing the qualitatively different behavior.

In terms of the behavior of contacts, we confirm that a larger proportion of the total contacts are weak, while the proportion of sliding contacts out of the total contacts is less than $45 \%$.
More importantly, we find less than $50 \%$ weak sliding contacts with respect to the total number of weak contacts. On the other hand, the proportion of weak sliding contacts with respect to the total sliding contacts is significantly higher. The latter is in agreement with earlier studies that show that friction is more highly mobilized in weak contacts [50,53].

As a consequence of the isotropic initial configuration, we find that the directional distribution of normal forces at the initial state are isotropic for all $\mu$. At maximum compression, we observe a higher mean, a lower peak, and a wider tail of the force distribution in the compressive $z$ direction, while the distributions in the two lateral directions remain identical, narrower, with a shorter tail. Due to history, after uniaxial compression and tension, the initial states are not recovered when the original state, $\varepsilon_{\mathrm{dev}}=0$, is reached. Forces are weaker and one has fewer contacts in the tensile $z$ direction.

We have also presented two averaging methods for the polar representation of contacts using the constant azimuthal and constant height methods. For our data, a second order tensor is insufficient to describe the structural anisotropy. We find a sixth order distribution with two peaks leading to three anisotropy state descriptors $\left(\epsilon_{2}, \epsilon_{4}\right.$, and $\left.\epsilon_{6}\right)$.

The second harmonic $\epsilon_{2}$ is close to $F_{\mathrm{dev}}^{s} / \sqrt{3}$, so that for different friction, the maximum $\epsilon_{2}$ values behave in a similar fashion to the maximum deviatoric fabric. A second order tensorial descriptor is sufficient for the normal force but the tangential force and mobilized friction show a similar behavior to the contact distribution-requiring a higher order harmonic approximation due to the two strong peaks at $\pi / 4$ and the dip around $\pi / 2$, i.e., the microstructural and force features that kick in at nonaxial different directions.

Future studies should concern exploring higher order tensors and the validity of the findings for other nonisotropic deformation modes (e.g., under simple and pure shear or triaxial tests). Furthermore, recent experiments [23] will allow us to validate the present observations from DEM. The final goal is to develop constitutive models of particulate matter based on the microscopic insights gained [58], and apply those, e.g., to cyclic loading as relevant in many applications such as, e.g., roads.

\section{ACKNOWLEDGMENTS}

Helpful discussions with N. Kumar, A. Singh, V. Ogarko, F. Radjai, and J. N. Roux are gratefully acknowledged. This work was financially supported by the European Union funded Marie Curie Initial Training Network, FP7 (ITN-238577); see [59] for more information.

\section{APPENDIX A: AVERAGING METHODS}

In this Appendix, we describe the two averaging methods, namely the constant azimuthal angle method $(b)$ and the constant height method $(h)$.

\section{Constant azimuthal angle (bin width) method}

Given the three normal unit vector components $\hat{n}_{x}, \hat{n}_{y}$, and $\hat{n}_{z}$ for each contact pair, to calculate the azimuthal angle, one needs the polar orientation $\arccos \left(\hat{n}_{z}\right)$ of the normal unit vector in the direction relative to the active (axial) direction 


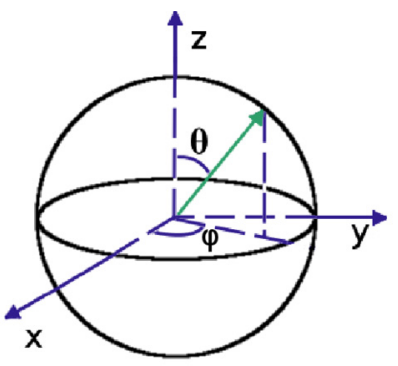

(a)

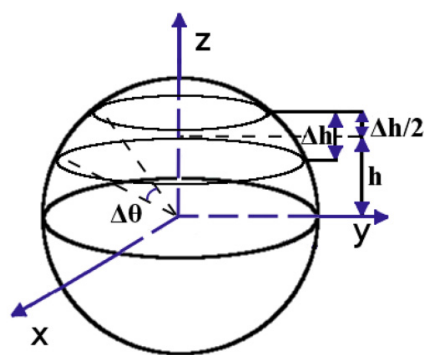

(b)
FIG. 18. (Color online) Schematic representation of the angles of the contact unit vector (green arrow) for (a) the constant bin width $\Delta \theta$ method and (b) the constant height method. The angles $\theta$ and $\varphi$ are the azimuthal angle and polar angle of the system, respectively.

as schematically described in Fig. 18(a). We average over the spherical azimuthal (vs polar) $(r, \varphi)$ coordinate and then distribute the vectors, based on their orientation into bins of width $\Delta \theta$.

The fraction of contacts in a single bin is defined as $\phi^{\theta}=C^{\theta} / C_{\text {tot }}$, where $C^{\theta}=\sum_{C \in b} 1$ and $b \in[\theta-\Delta \theta / 2 ; \theta+$ $\Delta \theta / 2]$. Furthermore, $\phi^{\theta}$ is normalized with the surface of the spherical annulus for each $b$ by the factor $\Delta \theta \sin \theta$ to yield the azimuthal contact probability density $P(\theta)=\left(\phi^{\theta} / \Delta \theta \sin \theta\right)$ such that $\int_{0}^{\pi} P(\theta) \sin \theta \Delta \theta=1$. $^{2}$ The polar distributions of the normal forces, tangential forces, and mobilized friction are given, respectively, by $f_{n}(\theta)=\left(\sum_{C \in b} f_{n}\right) /\left(C^{\theta}\right), f_{t}(\theta)=$ $\left(\sum_{C \in b} f_{t}\right) /\left(C^{\theta}\right)$, and $\psi_{t}(\theta)=\left[\sum_{C \in b}\left(f_{t} / \mu f_{n}\right)\right] /\left(C^{\theta}\right)$, where the normalization with the number of contacts in each bin has been used.

\section{Constant height method}

In the constant height method, we sort the vectors based on their orientations into $n$ azimuthal spherical segments with equal heights $\Delta h=\cos \theta_{2}-\cos \theta_{1}$ as shown schematically in Fig. 18(b). Given the polar radius $r$, and the height from the center of each segment to the middle of the sphere $h$, the polar angle $\theta$ of each vector is calculated for every $\hat{n}_{z} \in h$. The fraction of contacts within each segment range is then

\footnotetext{
${ }^{2}$ An alternative to the $\Delta \theta \sin \theta$ normalization is a discrete formulation $\cos \theta_{f}-\cos \theta_{i}$ where $\theta_{i}=\theta-\Delta \theta / 2$ and $\theta_{f}=\theta+\Delta \theta / 2$.
}

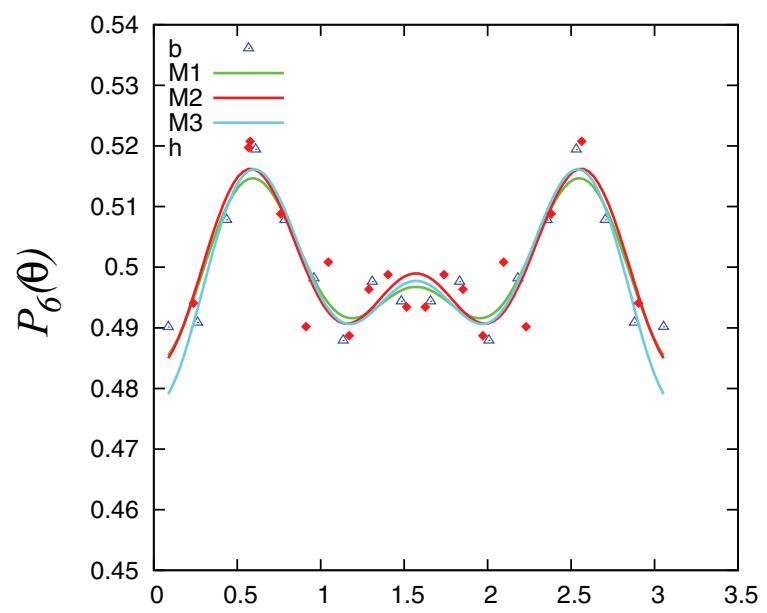

FIG. 19. (Color online) Sixth order fits of methods 1, 2, and 3 to the $P(\theta)=\left(\phi^{\theta} / \Delta \theta \sin \theta\right)$ data at dimensionless time $\tau=0.076$. The solid red symbols represent data obtained using the constant height method $(h)$, while the triangles are those obtained with the constant bin width method $(b)$.

given as $\phi^{h}=C^{h} / C_{\text {tot }}$, where $C^{h}=\sum_{C \in h} 1$ and $h \in[h-$ $\Delta h / 2 ; h+\Delta h / 2]$. With $-1 \leqslant h \leqslant 1$ specifying the number of bins $M_{h}$ (e.g., $M_{h}=20$ ) allows us to compute all $h$ intervals and boundaries.

Other quantities, including the normal and tangential forces and mobilized friction, can be computed similarly to the constant bin width method, just by summation and normalization with $C^{h}$ instead of $C^{\theta}$.

\section{APPENDIX B: FIT METHODS}

In the following, we describe different methods of obtaining the anisotropy state descriptors using the data obtained using the constant bin width or the constant height methods.

\section{Method 1: Fit azimuthal contact probability density $P(\theta)=\left(\phi^{\theta} / \Delta \theta \sin \theta\right)$}

In the first case, we fit the azimuthal contact probability density data $P(\theta)=\left(\phi^{\theta} / \Delta \theta \sin \theta\right)$ using the harmonic equation (25). Note that for the special case of uniaxial compression, Eq. (24) does not lead to consistent results across the methods and is thus disregarded. However, as shown

TABLE IV. Selected references on the orientational contact distribution for various modes. For an isotropic sample, $K=C / 4 \pi$ and $C$ is the coordination number.

\begin{tabular}{|c|c|c|c|c|}
\hline & Reference & Mode & $\begin{array}{c}\text { Contact } \\
\text { probability } P(\theta)\end{array}$ & Harmonic function \\
\hline 1. & Azema et al. [55] & triaxial & second order & $\frac{1}{4 \pi}\left[1+\epsilon\left(3 \cos ^{2} \theta-1\right)\right]$ \\
\hline 2. & Deng and Dave [60] & $\begin{array}{l}\text { particle settling in a } \\
\text { cylindrical geometry }\end{array}$ & higher order & \\
\hline 3. & Ishibashi et al. [61] & triaxial & second order & $K\left(1-\epsilon+3 \epsilon \cos ^{2} \theta\right)$ \\
\hline 4. & Jenkins [62] & triaxial & second order & $\frac{C}{4 \pi}\left[(1-\epsilon)+3 \epsilon \cos ^{2} \theta\right]$ \\
\hline 5. & Silbert et al. [63] & $\begin{array}{l}\text { 3D particle settling } \\
\text { on a flat base }\end{array}$ & higher order & \\
\hline 6. & Staron and Radjai [50] & 2D avalanche & higher order & \\
\hline
\end{tabular}




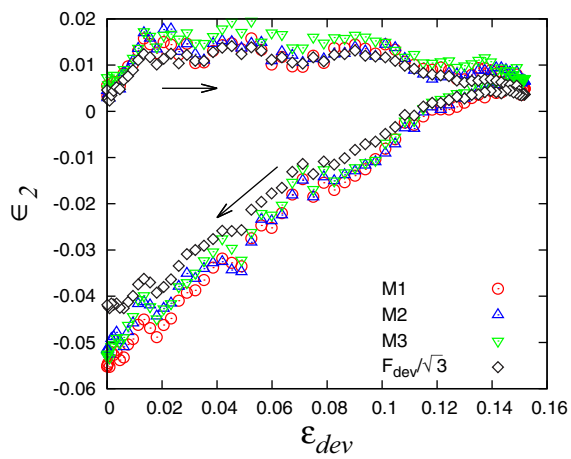

(a)

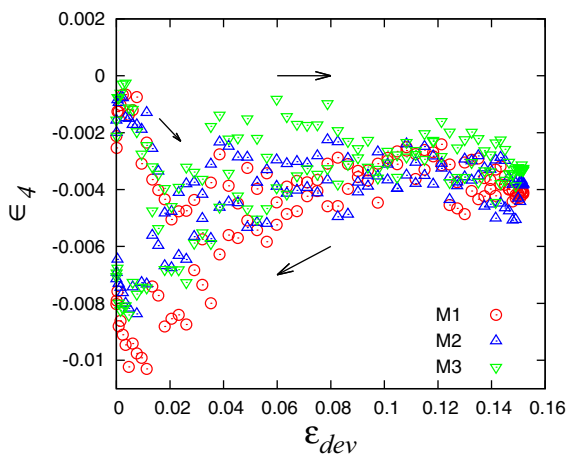

(b)

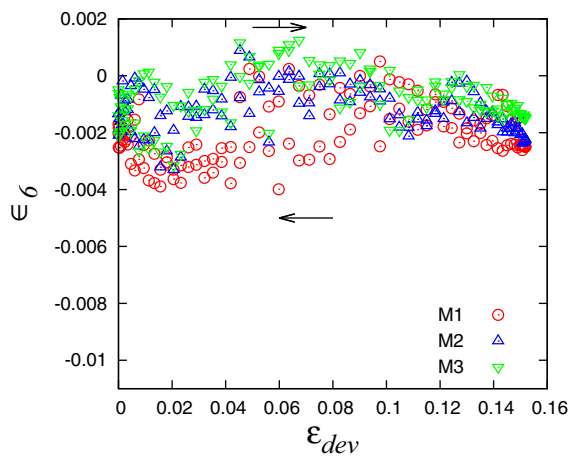

(c)

FIG. 20. (Color online) Evolution of the anisotropy state descriptors $\epsilon_{2}, \epsilon_{4}$, and $\epsilon_{6}$ of the sixth order expansion Eq. (25) as function of deviatoric strain using the three methods for $\mu=0$.

in different literature especially under triaxial compression, the second order approximation $P_{2}(\theta)$ is sufficient to fully capture the contact probability density data. Exemplary (not exhaustive) references of works where forms of Eq. (24) as applied to various triaxial tests are presented in Table IV. Other experimental and numerical setups are also shown. Note that in these cases, the orientational contact distribution obtained is not of second order and are not fitted.

\section{Method 2: Fit to the constant height data}

In the second case, we directly fit the fraction of contacts $\phi^{h}$, generated using the constant height method such that the bad statistics at the poles are not overexposed, as in Method 1. In this case, we set the zero order parameter $a_{0}=0.5$.

\section{Method 3: Fit to fraction of contacts $\phi^{\theta}$ with a $\Delta \theta \sin \theta$ scaling}

In the third case, we fit the fraction of contacts $\phi^{\theta}$ data directly using harmonic Equations (24) or (25) multiplied by $\Delta \theta \sin \theta$. The original signal is a first order sinus, i.e., less weighting is given to the areas close to the poles such that their larger statistical errors are suppressed.

In Fig. 19, we show the sixth order harmonic fits using methods 1 (M1), 2 (M2), and 3 (M3) to the constant bin width (b) and constant height $(h)$ data for $\mu=0$. For each method, three anisotropy state descriptors, namely $\epsilon_{2}, \epsilon_{4}$, and $\epsilon_{6}$ are obtained. Here, as an example, we show a single snapshot, namely at $\tau=0.076$. Note that the original data are from 0 to $\pi / 2$ and the extension from $\pi / 2$ to $\pi$ is only a mirror image. Focusing on the numerical data (symbols), we observe two strong peaks at about $\pi / 2 \pm \pi / 4$ and a local maximum at $\pi / 2$. The twin peaks indicate that a distribution higher than second order is needed. The data are well captured by a sixth order approximation $P_{6}(\theta)$ (solid lines). Comparing the $b$ and $h$, we observe stronger scatter at the boundaries for the $b$ data due to the weak statistics at the extreme $\theta$ values $(0$ and $\pi)$. For the fits, we observe that M1, M2, and M3 are close and the major differences between them are most pronounced at both peaks and extrema.

In Fig. 20, we plot the evolution of the anisotropy state descriptors $\epsilon_{2}, \epsilon_{4}$, and $\epsilon_{6}$ as functions of the deviatoric strain and compare the three methods. From Fig. 20(a), during loading, $\epsilon_{2}$ grows and reaches a maximum at $\varepsilon_{\mathrm{dev}} \approx 0.025$ from where it slightly decreases. After maximum loading, $\epsilon_{2}$ decreases (taking well into account the sign change) and becomes increasingly negative until it reaches $\epsilon_{2} \approx-0.055$ at complete unloading $(\tau=1)$. Comparing the three methods, M3 is slightly off (higher) during loading while M1 is also slightly off at the end of unloading. Interestingly, we find that the evolution of $\epsilon_{2}$ is similar to the simple definition of the deviatoric fabric in Eq. (19) involving a difference between the fabric component of the axial direction and the average of the components of the two lateral components. Note that the definition of the fabric used here is based on Eq. (16) which considers only the contacts and not the dependence on the volume fraction. We note that the magnitude of $\epsilon_{2}$ is proportional to $F_{\text {dev }} / \sqrt{3}$ [black diamonds in Fig. 20(a)] with M3 slightly off during loading and the unloading data also showing slight variations. From Figs. 20(b) and 20(c), the values of $\epsilon_{4}$ and $\epsilon_{6}$ appear small compared to $\epsilon_{2}$ but must not be neglected. When $\epsilon_{2}$ is taken as the structural anisotropy state descriptor, it much resembles $F_{\mathrm{dev}}$. However, the higher order anisotropy is quantified by the $\epsilon_{4}$ (which is negative) and $\epsilon_{6}$ (which is strongly fluctuating and different for different methods).
[1] F. Alonso-Marroquin and H. J. Herrmann, Phys. Rev. Lett. 92, 054301 (2004).

[2] O. I. Imole, M. Wojtkowski, V. Magnanimo, and S. Luding, Powd. \& Grains 1542, 601 (2013).

[3] S. Luding, Gran. Mat. 10, 235 (2008).

[4] GDR MiDi, Eur. Phys. J. E 14, 341 (2004).

[5] J. Schwedes, Gran. Mat. 5, 1 (2003).
[6] X. Z. An, R. Y. Yang, R. P. Zou, and A. B. Yu, Powd. Technol. 188, 102 (2008).

[7] S. J. Antony and N. P. Kruyt, Phys. Rev. E 79, 031308 (2009).

[8] S. J. Antony and M. A. Sultan, Phys. Rev. E 75, 031307 (2007).

[9] O. Durán, N. P. Kruyt, and S. Luding, Int. J. Solids Struct. 47, 2234 (2010). 
[10] L. E. Silbert, D. Ertas, G. S. Grest, T. C. Halsey, and D. Levine, Phys. Rev. E 65, 031304 (2002).

[11] M. M. Bandi, M. K. Rivera, F. Krzakala, and R. E. Ecke, Phys. Rev. E 87, 042205 (2013).

[12] T. S. Majmudar and R. P. Behringer, Nature (London) 435, 1079 (2005).

[13] J. Zhang, T. Majmudar, A. Tordesillas, and R. Behringer, Gran. Mat. 12, 159 (2010)

[14] C. M. Wensrich, E. H. Kisi, J. F. Zhang, and O. Kirstein, Gran. Mat. 14, 671 (2012)

[15] F. Radjai, D. E. Wolf, M. Jean, and J. J. Moreau, Phys. Rev. Lett. 80, 61 (1998).

[16] C. Thornton and S. J. Anthony, Philos. Trans. R. Soc. London Ser. A 356, 2763 (1998).

[17] F. Radjaï, S. Roux, and J. J. Moreau, Chaos 9, 544 (1999).

[18] A. S. J. Suiker and N. A. Fleck, J. Appl. Mech. 71, 350 (2004).

[19] I. Ishibashi, J. T. Jenkins, and J. W. Choi, Soils \& Found. 36, 45 (1996).

[20] O. I. Imole, N. Kumar, V. Magnanimo, and S. Luding, KONA Powd. Part. J 30, 84 (2013).

[21] A. Ezaoui and H. Di Benedetto, Géotechnique 59, 621 (2009).

[22] M. van Hecke, J. Phys.: Condens. Matter 22, 033101 (2009).

[23] N. Brodu, J. Dijksman, and R. P. Behringer (private communication).

[24] S. Luding, Int. J. Solids Struct. 41, 5821 (2004).

[25] S. Luding and E. Bauer, in International Symposium on Geomechanics and Geotechnics Shanghai Conference Proceedings (CRC Press/Balkema, Netherlands, 2010), pp. 495-499.

[26] V. Ogarko and S. Luding, J. Chem. Phys. 136, 124508 (2012).

[27] A. Kwade, D. Schulze, and J. Schwedes, Powd. Hand. \& Proc. 6, 61 (1994).

[28] H. Zetzener and J. Schwedes, in Proceedings of the 6th International Conference on Handling and Transportation, Brisbane, Australia, edited by P. Wypych (Curran Associates, Inc., Red Hook, NY, 1998), pp. 51-55.

[29] A. Kwade, D. Schulze, and J. Schwedes, Powd. Hand \& Proc. 6, 199 (1994).

[30] F. Göncü, O. Duran, and S. Luding, C. R. Mécanique 338, 570 (2010).

[31] C. Thornton and L. Zhang, Philos. Mag. 86, 3425 (2006).

[32] S. Luding, J. Phys. Condens. Matter 17, S2623 (2005).

[33] T. Weinhart, R. Hartkamp, A. R. Thornton, and S. Luding, Phys. Fluids 25, 070605 (2013).

[34] T. Weinhart, A. R. Thornton, S. Luding, and O. Bokhove, Gran. Mat. 14, 289 (2012).

[35] M. Madadi, O. Tsoungui, M. Latzel, and S. Luding, Int. J. Solids Struct. 41, 2580 (2004).

[36] S. Luding, Part. Sci. Technol. 26, 33 (2007).

[37] M. R. Shaebani, M. Madadi, S. Luding, and D. E. Wolf, Phys. Rev. E 85, 011301 (2012).
[38] L. La Ragione and V. Magnanimo, Phys. Rev. E 85, 031304 (2012).

[39] M. Oda, Soils \& Found. 1, 17 (1972).

[40] M. Satake, in Proceedings of the IUTAM Symposium on Deformation and Failure of Granular Materials (Balkema, Delft, The Netherlands, 1982).

[41] F. Göncü and S. Luding, Acta Geotech. 8, 629 (2013).

[42] N. Kumar, O. I. Imole, V. Magnanimo, and S. Luding, Particuology 12, 64 (2014).

[43] P. J. Digby, J. Appl. Mech. 48, 803 (1981).

[44] V. Magnanimo, L. La Ragione, J. T. Jenkins, P. Wang, and H. A. Makse, Europhys. Lett. 81, 34006 (2008).

[45] K. Walton, J. Mech. Phys. Solids 35, 213 (1987).

[46] N. Kumar, O. I. Imole, V. Magnanimo, and S. Luding, in Powders and Grains, edited by A. Yu, K. Dong, R. Yang, and S. Luding, AIP Conf. Procs. No. 1542 (AIP, NY, 2013), pp. 1238-1241.

[47] R. Tykhoniuk, J. Tomas, S. Luding, M. Kappl, L. Heim, and H. J. Butt, Chem. Eng. Sci. 62, 2843 (2007).

[48] N. P. Kruyt and L. Rothenburg, Int. J. Solids Struct. 40, 3537 (2003).

[49] N. Guo and J. Zhao, Comp. Geotech. 47, 1 (2013).

[50] L. Staron and F. Radjai, Phys. Rev. E 72, 041308 (2005).

[51] E. Azéma and F. Radjai, Phys. Rev. E 81, 051304 (2010).

[52] E. Azéma, F. Radjai, R. Peyroux, and G. Saussine, Phys. Rev. E 76, 011301 (2007).

[53] L. Staron, F. Radjai, and J. Vilotte, J. Stat. Mech. (2006) P07014.

[54] N. P. Kruyt and L. Rothenburg, Int. J. Solids Struct. 39, 571 (2002).

[55] E. Azéma, F. Radjai, and G. Saussine, Mech. Mater. 41, 729 (2009).

[56] H. J. Bunge and P. R. Morris, Texture Analysis in Materials Science (Butterworth, London, 1982).

[57] F. Radjai, J. Y. Delenne, E. Azéma, and S. Roux, Gran. Mat. 14, 259 (2002).

[58] N. Kumar, V. Magnanimo, and S. Luding, Acta Mechanica, doi:10.1007/s00707-014-1155-8.

[59] http://www.pardem.eu/.

[60] X. L. Deng and R. N. Davé, Granular Matter 15, 401 (2013).

[61] I. Ishibashi, T. Agarwal, and S. A. Ashraf, in Proceedings of the First U.S. Conference on Discrete Element Methods, Colorado School of Mines, Golden, CO., edited by G. G. Mustoe, M. Henriksen, and H.-P. Huttelmaier (Colorado School of Mines, Colorado, 1989), Vol. 23, p. 12.

[62] J. T. Jenkins, in Micromechanics of Granular Materials, edited by M. Satake and J. T. Jenkins (Elsevier, Amsterdam, 1988), pp. 245-252.

[63] L. E. Silbert, G. S. Grest, and J. W. Landry, Phys. Rev. E 66, 061303 (2002). 